\title{
Morphological, Physiological, and Molecular Responses of Sweetly Fragrant Luculia Gratissima During the Floral Transition Stage Induced by Short- day Photoperiod
}

\section{Xiongfang Liu}

Chinese Academy of Forestry https://orcid.org/0000-0002-7842-5717

Youming Wan

Chinese Academy of Forestry

Jing An

Chinese Academy of Forestry

\section{Xiujiao Zhang}

Chinese Academy of Forestry

\section{Yurong Cao}

Chinese Academy of Forestry

Zhenghong Li ( $\square$ luculia_gratissima@163.com )

Research Institute of Resources Insects, Chinese Academy of Forestry, 650224 Kunming, China https://orcid.org/0000-0003-1677-4628

\section{Xiuxian Liu}

Chinese Academy of Forestry

\section{Hong Ma}

Chinese Academy of Forestry

\section{Research article}

Keywords: Luculia gratissima, Floral transition, Photoperiod, Flowering pathway, Phytohormone, Regulatory network

Posted Date: October 19th, 2020

DOl: https://doi.org/10.21203/rs.3.rs-92695/v1

License: (c) (i) This work is licensed under a Creative Commons Attribution 4.0 International License. Read Full License 


\section{Abstract}

Background: Photoperiod-regulated floral transition is vital to the flowering plant. Luculia gratissima 'Xiangfei' is a flowering ornamental plant with high development potential and is a short-day woody perennial. However, the genetic regulation of short-day-induced floral transition in L. gratissima is unclear. To systematically research the responses of $L$. gratissima during this process, dynamic changes in morphology, physiology, and transcript levels were observed and identified in different developmental stages of long-day and short-day-treated shoot apexes.

Results: The results showed that floral transition in L. gratissima occurred $10 \mathrm{~d}$ after short-day induction, but flower bud differentiation did not occur under long-day conditions. A total of 1,226 differentially expressed genes were identified, of which 146 genes were associated with flowering pathways of sugar, phytohormones, photoperiod, ambient temperature, and aging signals, as well as floral integrator and meristem identity genes. The trehalose-6-phosphate signal positively modulated floral transition by interacting with SPL4 in the aging pathway. Endogenous gibberellin, abscisic acid, cytokinin, and jasmonic acid promoted floral transition, whereas strigolactone inhibited it. In the photoperiod pathway, FD, COL12, and NF-Ys positively controlled floral transition, whereas PRR7, FKF1, and LUX negatively regulated it. SPL4 and pEARLI1 positively affected floral transition. SOC1 and AGL24 integrated multiple flowering signals to modulate the expression of FUL/AGL8, AP1, LFY, SEPS, SVP, and TFL 1, thereby regulating floral transition. Finally, we propose a regulatory network model for short-day-induced floral transition in L. gratissima.

Conclusions: Short-day photoperiod activated systemic responses of morphology, physiology, and transcript levels in L. gratissima and induced the generation of floral transition signals in the photoperiod pathway. Furthermore, multiple flowering signal pathways including phytohormone-, sugar-, temperature, age-related genes synergistically control this process. This study improves our understanding of flowering time regulation in L. gratissima and provides knowledge for its production and commercialization.

\section{Background}

Floral transition (the switch from vegetative to reproductive development) is a critical stage in life history of flowering plants, particularly in horticultural ornamental plants $[1,2]$. This process is regulated by both environmental and endogenous signals [1]. Recently, major breakthroughs have been made in research on the molecular regulatory networks of floral transition in Arabidopsis thaliana [3, 4]. In A. thaliana, different endogenous (autonomous, gibberellin, circadian rhythm, age, sugar signals) and environmental (vernalization, temperature, and photoperiod) signals congregate on some floral integrators, such as SUPPRESSOR OF OVEREXPRESSION OF CONSTANS 1 (SOC1), FLOWERING LOCUS T(FT), and AGAMOUSLIKE24 (AGL24), further activating floral meristem identity genes, such as LEAFY (LFY) and APETALA1 (AP1), which irreversibly convert vegetative meristem to floral meristem [5]. However, there is still much to learn regarding the regulation of floral transition in perennial woody plants. Perennial woody 
plants do not die after flowering. Instead, they produce new flower buds and vegetative branches annually and have characteristics of long reproductive cycles and seasonal flowering [6]. Therefore, studies on annual plants cannot completely reveal the floral transition mechanisms in perennial woody plants. There are significant differences in the molecular mechanisms of floral transition in perennial woody plants compared with those of $A$. thaliana. For example, gibberellin (GA) promotes the transition from vegetative to reproductive development in $A$. thaliana but has inhibitory effects in some perennial woody plants [79]. Therefore, it is particularly important to study the molecular mechanisms of floral transition in perennial woody plants.

Luculia gratissima (Wall.) Sweet. (Rubiaceae) is a perennial evergreen shrub or small tree that is distributed in the south-eastern edge of the Tibetan plateau in southwest China and neighboring Nepal and Myanmar [10]. L. gratissima 'Xiangfei', a new cultivar cultivated by our research team for many years, has pink flowers, a strong fragrance, and a large and dense inflorescence (Fig. 1a, b); it is a woody horticultural flower with great ornamental value and development potential. However, this plant has not entered the large-scale commercial production stage because of imperfect flowering time regulation techniques. Previous studies showed that the cultivar 'Xiangfei' can only complete floral transition at short-day (SD) photoperiods [11], and thus controlling daylength to induce flowering is required to achieve year-round production. Therefore, understanding the mechanisms of short-day-induced floral transition in L. gratissima 'Xiangfei' has important significance for understanding and solving flowering-related problems.

In the present study, we investigated responses of L. gratissima during short-day-induced floral transition stage at the morphological, physiological, and transcriptome levels. The aims of this study were: (1) to observe shoot apexes of $L$. gratissima of short-day treatment during five developmental stages using morphological and histological methods to identify the time point of floral transition in L. gratissima; (2) to measure endogenous substance contents to study the soluble sugar and hormone effects in floral transition in L. gratissima; (3) to conduct an RNA sequencing (RNA-seq) analysis of the transcriptomes of L. gratissima shoot apexes at four different stages, 7, 10, 13, and 19 days after the initiation of long-day (LD) and short-day (SD) treatments, to study the molecular regulatory mechanism of short-day-induced floral transition in L. gratissima. The results presented in this research will aid in regulating L. gratissima flowering and achieving year-round production. Additionally, identification of important regulatory genes will provide important guidance for flowering-related molecular breeding in the future.

\section{Results}

\section{Morphological differentiation of shoot apexes during floral transition}

The morphological differentiation of L. gratissima shoot apexes was observed through paraffin sections. The results showed that $0 \mathrm{~d}$ to $7 \mathrm{~d}$ under the SD treatment (SD0 to SD7) was the vegetative growth stage (undifferentiated stage), in which the tip of the growth cone in the bud was narrow and pointed and surrounded by leaf primordia (Fig. 2a-c). At $10 \mathrm{~d}$ after the initiation of the SD treatment (SD10), the bract 
primordial differentiation stage began in which the growth cone of the bud appeared hemispherical, which was an important marker of the transition from vegetative growth to reproductive growth. Subsequently, the two sides of the hemispherical growth cone elongated to form two protrusions, i.e., bract primordia (Fig. 2d-f). At $13 \mathrm{~d}$ after the initiation of the SD treatment (SD13), the inflorescence primordial differentiation stage began. At this stage, the growth cone in the bract primordia elongated to form three hemispherical protrusions, i.e., inflorescence primordia. Simultaneously, the lateral base of the bract primordia differentiated into lateral inflorescence primordia. Next, bilateral protrusions at each hemispherical inflorescence primordium differentiated into bract inflorescences (Fig. $2 \mathrm{~g}-\mathrm{i}$ ). At $19 \mathrm{~d}$ after the initiation of the SD treatment (SD19), the floret primordial differentiation stage began and a single inflorescence primordium in the bract primordia gradually widened to become floret primordia at the tip of the bud (Fig. 2j-I). These results showed that the floral transition period began $10 \mathrm{~d}$ after the initiation of the SD treatment, and the selection of time points before and after this period could facilitate the physiological study of floral transition. Therefore, the LD treatment was used as a control in this study, and $7 \mathrm{~d}$ (SD7), $10 \mathrm{~d}$ (SD10), $13 \mathrm{~d}$ (SD13), and $19 \mathrm{~d}$ (SD19) in the SD treatment were selected to study the physiological and molecular regulation patterns of floral transition.

\section{Dynamic changes in endogenous substance content during floral transition}

Contents of soluble sugars and endogenous hormones (gibberellin [ $\mathrm{GA}_{3}$ ], indoleacetic acid [IAA], abscisic acid [ABA], and zeatin [ZT]) were measured at $0 \mathrm{~d}$ [SD0], $7 \mathrm{~d}$ [SD7], $10 \mathrm{~d}$ [SD10], $13 \mathrm{~d}$ [SD13], and $19 \mathrm{~d}$ [SD19] after the initiation of the SD treatment. The Kruskal-Wallis $\mathrm{H}$ test results showed that except for $\mathrm{GA}_{3}$, which could not be detected because it was below the limit of quantitation $(0.1 \mathrm{ng} / \mathrm{ml})$, there were significant differences in the contents of the other substances among the five stages (adjusted $p<0.05$; Fig. 3). Soluble sugar, ZT, and IAA reached their peaks at SD0, which were $28.86 \pm 0.67 \mathrm{mg} \mathrm{g}^{-1} \mathrm{FW}, 2.15 \pm$ $0.30 \mathrm{ng} \mathrm{g}^{-1} \mathrm{FW}$, and $0.69 \pm 0.04 \mathrm{ng} \mathrm{g}^{-1} \mathrm{FW}$, respectively. Additionally, soluble sugar and ZT decreased from SD0 to SD19. Interestingly, IAA showed an increase in SD13 before decreasing. Similarly, ABA initially increased from SD0 to SD13 and subsequently declined.

Additionally, post-hoc results showed that there were extremely significant differences in the pairwise comparisons between the five timepoints for ABA $(p<0.001)$. IAA only showed no significant differences between SD7 and SD13 $(p>0.1)$. Soluble sugar did not show any significant differences between SD0 and SD7 $(p>0.1)$. ZT did not show any significant differences between SD0 and SD10, between SD7 and SD10, or between SD13 and SD19 $(p>0.1)$. From these results, it can be seen that ABA levels changed rapidly and dynamically over these five stages, whereas ZT levels exhibited little change over this same time period. Changes in soluble sugar content mainly occurred in later periods (SD13 to SD19). In contrast to these substances, IAA changes were relatively constant (Fig. 3).

\section{RNA-seq and qRT-PCR identification of differentially expressed genes (DEGs)}

Transcriptomes were generated for three biological replicates from the SD and LD treatments at each of the time points corresponding to the four stages of bud differentiation in SD treatment plants, i.e., at $7 \mathrm{~d}$, 
$10 \mathrm{~d}, 13 \mathrm{~d}$, and $19 \mathrm{~d}$ (Fig. 2), yielding a total of 24 transcriptomes. A total of 1,236,426,670 raw sequencing reads were generated from 24 samples, $1.2 \times 10^{9}$ high-quality clean reads $(181 \mathrm{~Gb})$ were obtained after filtering, with mean Q20, Q30, and GC contents of $99.11 \%, 97.18 \%$, and $43.53 \%$, respectively (Additional file 1: Table S1). A total of 79,870 unigenes ( $\geq 200 \mathrm{~b}$ ) were generated from de novo assembly, and the $\mathrm{N} 50$ length was 2,118 bp (Additional file 2: Table S2). Among these unigenes, 35,725 unigenes (44.73\%) were successfully annotated to at least one database (Additional file 3: Fig. S1).

With RNA from the same 24 samples used for transcriptome generation, qRT-PCR was conducted for nine flowering-related unigenes identified in through RNA-seq, including COP1 (Unigene0031506), ZTL (Unigene0041339), FKF1 (Unigene0038380), GI (Unigene0051409), ELF3 (Unigene0051761), PRR1 (Unigene0045946), PRR7 (Unigene0003564), PRR5 (Unigene0047475), and LHY (Unigene0035686). The results of qRT-PCR showed that except for PRR5 (Unigene0047475), the expression patterns of the other eight genes were generally consistent with the RNA-seq data (Additional file 4: Fig. S2), indicating that the transcriptome data generated in this study were reliable and valid.

A total of 113 (LD7-vs.-SD7), 420 (LD10-vs.-SD10), 483 (LD13-vs.-SD13), and 464 (LD19-vs.-SD19) differentially expressed genes (DEGs) were obtained by comparing the LD and SD treatments (Additional file 5: Fig. S3; Additional file 6: Table S3). A total of 1,226 DEGs were identified from these four comparisons, of which five DEGs were shared by four comparisons, and 250 DEGs were present in at least one comparison. There were 110, 302, 288, and 276 stage-specific DEGs in LD7-vs.-SD7, LD10-vs.SD10, LD13-vs.-SD13, and LD19-vs.-SD19, respectively (Additional file 5: Fig. S3).

\section{Functional classifications of DEGs}

MapMan is an effective tool for systematic analysis of plant transcriptome metabolic pathways and other biological processes [12]. We employed MapMan to overview transcriptional changes in regulatory, metabolic, and cellular response-related genes (Additional file 7: Table S4). In "regulation overview", more DEGs were detected in the other three comparisons contrasted with LD7-vs.-SD7, showing that the physiological and molecular characteristics after flower bud differentiation (SD10, SD13, and SD19) were significantly different from that before flower bud differentiation (SD7). In the IAA metabolic subclass, more DEGs were upregulated in LD19-vs.-SD19 compared with LD7-vs.-SD7, LD10-vs.-SD10, and LD13vs.-SD13 (Additional file 8: Fig. S4). However, IAA content was the highest at SD0 and lowest on SD10 and continuously decreased from SD13 to SD19 (Fig. 3). Therefore, IAA was not a key factor mediating floral transition in L. gratissima. ABA metabolism-related DEGs were significantly upregulated in all four comparisons (Additional file 8: Fig. S4), and ABA levels were overall increasing in the process of floral transition (Fig. 3), demonstrating that ABA could promote floral transition in L. gratissima. In "minor $\mathrm{CHO}$ metabolism", trehalose biosynthesis-related DEGs were only upregulated in LD7-vs.-SD7 (Additional file 9: Fig. S5). "Cellular response overview" showed that more development-related DEGs were upregulated in LD10-vs.-SD10 compared with the other three comparisons (Additional file 10: Fig. S6), indicating that these DEGs promoted floral transition in L. gratissima. 


\section{Co-expression module analysis for DEGs}

Weighted gene co-expression network analysis (WGCNA) is a systems biology method for analyzing the correlation relationships between genes in multiple samples [13]. In this study, the results of WGCNA showed that 1,226 DEGs in eight samples were clustered in 11 different co-expression modules (labelled with different colors) (Fig. 4a). It is noteworthy that four out of 11 co-expression modules significantly correlated with a single sample ( $r>0.9, p<0.05$; Fig. 4b; Additional file 11: Table S5). For example, the largest module (black module) included 247 (20.15\%) SD19-specific DEGs (Fig. 4b; Additional file 11: Table S5a).

We further conducted GO enrichment analysis on 11 co-expression modules, and only the greenyellow module was not significantly enriched for any GO terms (Additional file 12: Table S6). Some GO terms were specifically identified in only a single module. For example, 120 specific $\mathrm{GO}$ terms were identified in the black module, which mainly involved signal transduction and negative regulation of metabolic processes, and 34 module-specific GO terms were identified in the brown module, which was mainly associated with growth and development (Additional file 12: Table S6). However, several G0 terms, including "response to organic substance" and "response to a stimulus", appeared in multiple modules (Additional file 12: Table S6), indicating possible module-gene interactions. Overall, the extensively enriched GO terms showed that floral transition in L. gratissima activated functional activities at the systems level.

The 11 modules were divided into seven categories based on the correlations between modules (Fig. 4c). The heatmap showed that there was a high correlation between the blue, magenta, pink, and tan modules, in which the genes were highly expressed in SD7 and SD10 (Fig. 4b, C), and were significantly enriched in multiple GO terms involving secondary metabolite biosynthesis, signal transduction, and regulation of developmental processes (Additional file 12: Table S6).

\section{Identification of DEG expression patterns associated withfloral transition in L. gratissima}

According to the above functional classifications and WGCNA of these DEGs, and flowering-related genes previously reported in model plants (such as $A$. thaliana) [5, 7], a total of 146 unigenes were identified as homologous genes related to floral transition in L. gratissima, involving several flowering pathways: sugar metabolism, hormone metabolism and signal transduction, photoperiod, ambient temperature, aging pathways, as well as floral integrator and floral meristem identity genes. Among these floral transition-related homologous genes, stage-specific DEGs, and common DEGs in LD7-vs.-SD7, LD10-vs.SD10, LD13-vs.-SD13, and LD19-vs.-SD19 are listed in Additional file 13: Table S7.

\section{The expression pattern of sugar signal-related homologs}

The sugar signal pathway, which responds to the sugar budget in plants, is one of the important pathways mediating the transition from vegetative to floral meristems [5]. A total of 29 (19.86\%) DEGs associated with sugar signal-related genes were identified, involving 23 sugar signal-related homologs. 
These genes expressed differently in different development stages of L. gratissima. For example, HEXOKINASE (HK) homologs (Unigene0044869 and Unigene0044870) were significantly upregulated in LD7-vs.-SD7 and LD13-vs.-SD13, and a BETA-GLUCOSIDASE 24 (BGLU24) homolog (Unigene0013088) was significantly upregulated in LD10-vs.-SD10. Meanwhile, Unigene0009721 and Unigene0041893, homologs of GALACTINOL SYNTHASE 2 (GOLS2) and RAFFINOSE SYNTHASE (RFS) participating in raffinose synthesis, were upregulated in LD7-vs.-SD7. In addition, TREHALOSE-6-PHOSPHATE SYNTHSE (TPS) homologs (Unigene0019787, Unigene0024389, Unigene0013555, Unigene0054604, Unigene0004913, and Unigene0062998) were upregulated at various stages, and SWEET16 homolog (Unigene0012661) was significantly upregulated in LD7-vs.-SD7 and LD10-vs.-SD10 (Fig. 5e; Additional file 14: Table S8). Hence, these genes may directly or indirectly participate in floral transition in $L$. gratissima.

\section{The expression patterns of phytohormone metabolism and signal transduction homologs}

Many studies have demonstrated that various phytohormones participate in the regulation of floral transition [7, 14-16]. A total of 20 (13.70\%) DEGs associated with phytohormone metabolism were identified, and these involved 16 phytohormone metabolism homologous genes and were related to nine phytohormone metabolism pathways. Among these genes, GIBBERELLIN 2-BETA-DIOXYGENASE 1 (GA2OX1) homologs (Unigene0030732) and GA2OX8 homologs (Unigene0073113), which are involved in GA metabolism, were significantly upregulated in LD10-vs.-SD10 and/or LD19-vs.-SD19. Meanwhile, Unigene0034382 (CYP707A1 homolog) and Unigene0042754 and Unigene0042755 (NCED1 homologs), respectively, encoding abscisic acid (ABA) 8'-hydroxylase 1 and nine-cis-epoxycarotenoid dioxygenase, were significantly upregulated in LD10-vs.-SD10. In addition, a homolog (Unigene0035296) of YUC4, encoding indole-3-pyruvate monooxygenase, which mediates auxin biosynthesis, was significantly upregulated in LD19-vs.-SD19. Additionally, genes encoding cytokinin (CK) dehydrogenase 7 (CKX7; Unigene0036599) and cytokinin dehydrogenase (CYP735A1; Unigene0029738) were significantly downregulated in LD19-vs.-SD19. CYTOCHROME P450 734A1 (CYP734A1) homolog (Unigene0036368), which participates in brassinolide (BR) biosynthesis, was upregulated in LD10-vs.-SD10 and LD19-vs.SD19; the jasmonate (JA) metabolism-related JASMONATE O-METHYLTRANSFERASE (JMT) homolog (Unigene0020912) and the salicylic acid (SA) metabolism-related UDP-GLYCOSYLTRANSFERASE 74F1 (UGT74F1) homolog (Unigene0004033) were downregulated in LD19-vs.-SD19. A homolog of CAROTENOID CLEAVAGE DIOXYGENASE 7(CCD7, Unigene0069349) involving in strigolactone (SL) biosynthesis, was also identified and showed significant downregulation in LD10-vs.-SD10 (Fig. 5c; Additional file 14: Table S8).

A total of $39(25.85 \%)$ DEGs associated with phytohormone signal transduction were identified and involved 30 phytohormone signal transduction homologs that were associated with signal transduction for nine hormones. Among these DEGs, GID1B homologs (Unigene0032780, Unigene0032781, and Unigene0063035), encoding a gibberellin receptor, were upregulated in LD10-vs.-SD10, whereas an RGL3 homolog (Unigene0071862), encoding a DELLA protein, was significantly downregulated in LD19-vs.SD19. The ABA signal transduction-related EID1-LIKE F-BOX PROTEIN 3 (EDL3) homolog 
(Unigene0018152) was upregulated in LD10-vs.-SD10, and SAUR71 homologs (Unigene0021953 and Unigene0025106), encoding auxin-responsive protein, were upregulated in LD13-vs.-SD13 and LD19-vs.SD19. Moreover, in the CK signaling pathway, homologs of AHPs (Unigene0034629, Unigene0004315, and Unigene0034630), encoding histidine-containing phosphotransfer protein, were highly expressed in SD10, SD13, and SD19, and an ARR6 homolog (Unigene0049441), encoding a two-component response regulator, was upregulated in LD19-vs.-SD19. In addition, a BR/1 homolog (Unigene0024976) in the BR signaling pathway was significantly upregulated in LD7-vs.-SD7; homologs of MYC4 (Unigene0009399) and TIFYS (Unigene0022959 and Unigene0019294) in the JA signaling pathway were upregulated in LD10-vs.-SD10; a DWARF14 (D14) homolog (Unigene0028658), participating in SL signal transduction, was upregulated in LD7-vs.-SD7 but downregulated in LD19-vs.-SD19 (Fig. 5d; Additional file 14: Table S8).

\section{Expression patterns of genes associated with photoperiod pathways}

The photoperiod flowering pathways in plants include the photosensory pathway, the circadian clock, and the systemic effector [17]. A total of 10 (6.84\%) photoperiod-related homologs were identified. Among these homologs, CHLOROPHYLL A-B BINDING PROTEIN (CAB40, Unigene0075619) was downregulated in LD19-vs.-SD19, whereas CONSTANS-LIKE 12 (COL12, Unigene0039617) and FD (Unigene0027311) were upregulated in LD13-vs.-SD13. Meanwhile, homologs of the FLAVIN-BINDING KELCH REPEAT F-BOX PROTEIN 1 (FKF1, Unigene0038380) and the PSEUDO-RESPONSE REGULATOR 7 (PRR7, Unigene0003564) were both downregulated in LD13-vs.-SD13, and a LUX homolog (Unigene0011585) was downregulated in LD10-vs.-SD10, whereas homologs of the NF-YS (Unigene0025001, Unigene0002375, and Unigene0033157) were upregulated at one or more stages (Fig. 5a; Additional file 14: Table S8).

\section{Expression patterns of genes associated with the ambient temperature pathway}

Plant responses to photoperiod and temperature are coupled $[18,19]$. The photoperiod-induced floral transition could also affect the expression of a series of ambient temperature-related genes in plants. We identified 28 (19.18\%) ambient temperature-related DEGs involving 18 homologs, primarily including the HEAT STRESS TRANSCRIPTION FACTORS (HSFS), HEAT SHOCK PROTEIN/ COGNATE (HSPS), and pEARL/1, most of which were highly expressed at several stages under LD (Fig. 5b; Additional file 14: Table S8).

\section{Expression patterns of aging pathway-related, floral integrator, and floral meristem identity genes}

The aging pathway is an endogenous flowering pathway in plants [20]. SQUAMOSA PROMOTERBINDING-LIKE PROTEIN 4 (SPL4) homologs (Unigene0024429 and Unigene0024430) in the aging pathway were upregulated in LD10-vs.-SD10, LD13-vs.-SD13, and LD19-vs.-SD19 (Fig. 5f; Additional file 14: Table S8). 
Floral integrators combine environmental and endogenous signals to mediate flowering in plants [5]. The floral integrator gene SUPPRESSOR OF OVEREXPRESSION OF CONSTANS 1 (SOC1) homologs

(Unigene0039572 and Unigene0039575) were upregulated in LD10-vs.-SD10, LD13-vs.-SD13, and LD19vs.-SD19, whereas the AGAMOUSLIKE24 (AGL24) homolog (Unigene0049016) was downregulated in LD19-vs.-SD19 (Fig. 5g; Additional file 14: Table S8).

Genetic networks regulating floral transition in plants ultimately activated floral meristem identity genes, thereby causing the transformation from vegetative to floral meristems [21]. A total of $15(10.27 \%)$ related DEGs were identified, involving nine floral meristem identity genes (Additional file 14: Table S8). Among these genes, homologs of AGL8/FUL ( $A G L 8$, also known as FUL; Unigene0019277, Unigene0004737, Unigene0042052, Unigene0042053, and Unigene0042058), APETALA 1 (AP1; Unigene0019278, Unigene0019279, and Unigene0031106), LEAFY (LFY; Unigene0030979 and Unigene0030980), and SEPALLATAs (SEPS; Unigene0000607, Unigene0034045, and Unigene0025130) were upregulated in one or more developmental stages, whereas homologs of SHORT VEGETATIVE PHASE (SVP, Unigene0049018) and TERMINAL FLOWER 1 (TFL1, Unigene0026727) were downregulated in LD19-vs.SD19, LD10-vs.-SD10, and LD13-vs.-SD13 (Fig. 5h; Additional file 14: Table S8).

\section{Co-expression network of floral transition-related genes}

A co-expression network constructed using 126 floral transition-related DEGs with edge weights $>0.1$ showed ten hub genes with great connectivity, including homologs of GLYCERALDEHYDE-3PHOSPHATEDEHYDROGENASE (GAPDH, Unigene0005846), AKR1B1 (Unigene0076531), PKM (Unigene0073914), ENOLASE1 (ENO1; Unigene0011083 and Unigene0011084), MED37E (Unigene0051600), L-LACTATE DEHYDROGENASE A CHAIN (LDHA, Unigene0009368), HSP83A (Unigene0031524), FUL (Unigene0042052), and SEP4 (Unigene0025130) (Additional file 15: Fig. S7). The genes with the highest network degree were GAPDH (Unigene0005846), AKR1B1 (Unigene0076531), and PKM (Unigene0073914), which participated in sucrose and starch catabolism (Additional file 14: Table S8).

\section{Discussion}

The timing of floral transition in plants is jointly regulated by internal and external environmental cues, of which photoperiod is one of the major environmental factors that affect floral transition in plants $[5,22]$. L. gratissima is a horticultural ornamental plant with high development potential, and therefore elucidating the molecular mechanism of its SD photoperiod-induced floral transition is important to its year-round production for commercial purposes. In this study, we conducted transcriptome sequencing of L. gratissima shoot apexes at four stages under LD and SD treatments. A total of 79,870 unigenes were obtained by de novo assembly, of which $49.02 \%$ were successfully annotated. Currently, there is no report on $L$. gratissima transcriptome assembly and our assembled and annotated transcriptome of $L$. gratissima provides a valuable genetic resource for breeding this species.

\section{Sugar signal mediates floral transition in L. gratissima}


Sugars are an important energy source and participate in floral transition in plants as important signaling molecules $[23,24]$. Leaves are the primary organ of sugar synthesis in plants, and shoot apical meristems (SAMs) are the sites of sugar mobilization and consumption, both of which form an important sourcesink unit [25]. Floral transition in plants is not only directly associated with sugar content from source and sink but is also regulated by sugar transport [26]. Previous studies have indicated that source-sink regulation could be achieved by the interaction between the bidirectional sugar transporter SWEET and the FT-like protein [27]. In this study, SWEET16 (Unigene0012661) was significantly upregulated in LD7vs.-SD7 and LD10-vs.-SD10 (Fig. 5e; Additional file 14: Table S8), indicating that SWEET participated in sucrose transport during floral transition in L. gratissima. However, soluble sugars in SAMs decreased from SD0 to SD19 (Fig. 3), which is not consistent with the expression profile of genes associated with sucrose metabolism. We speculated that SAM only synthesized limited levels of soluble sugar but SWEET16 (Unigene0012661) expression in SAMs was only high at SD7 and SD10, and its expression level decreased as SD treatment duration increased (Fig. 5e; Additional file 14: Table S8), subsequently causing a decrease in the rate of the sucrose transport from leaves to SAMs; this suggests that sucrose only acts as an energy source in floral transition in L. gratissima.

Trehalose-6-phosphate (T6P) is a component of the plant sugar signaling system and has important effects on flowering and development [28]. In A. thaliana, the T6P pathway in leaves induced the expression of the florigen gene $F T$ in the photoperiodic pathway to affect floral transition, whereas in SAMs, the expression of $S P L$ in the aging pathway was controlled by the T6P pathway to directly affect the expression of floral transition-related genes [29]. Therefore, the T6P pathway is an important signal that coordinates flowering induction. In this study, except for the T6P synthase homolog TPS (Unigene0013555) that was downregulated in LD19-vs.-SD19, other TPSs were upregulated at one or more stages during floral transition in L. gratissima (Fig. 5e; Additional file 14: Table S8), showing that TPS homologs participate in floral transition in L. gratissima and the T6P signaling pathway is significantly enhanced during floral transition. SPL4 was also highly expressed at SD10, demonstrating that T6P in L. gratissima SAM promoted floral transition by regulating SPL4 expression. HK acts as a catalytic enzyme to catalyze hexose phosphorylation, as well as a glucose signal sensor mediating the interaction between the glucose signaling pathway and the ABA signaling pathway to regulate plant development [30, 31]. In this study, HK homologs (Unigene0044869 and Unigene0044870) were upregulated in LD7-vs.-SD7 and LD13-vs.-SD13 (Fig. 5e; Additional file 14: Table S8). We speculate that HK mainly catalyzed hexose phosphorylation to provide an energy source for initiating floral transition at SD7 and acted as a glucose signal sensor to participate in L. gratissima flower development at SD13.

In summary, the sugar metabolism-related genes TPS and HK entered the flowering regulatory network through the sugar signaling and hormone signaling pathways to regulate floral transition in L. gratissima.

\section{Phytohormones regulate floral transition in L. gratissima}

Phytohormones play important regulatory roles in plant development and the mechanisms of their participation in floral transition in many plants are extensively studied [7, 14-16]. However, the complex 
hormone regulatory network of floral transition in perennial woody plants remains unclear. We studied the regulatory patterns of hormones that participate in floral transition in L. gratissima.

As one of the most important phytohormones, the function of GA in regulating floral transition is mainly achieved through maintaining GA homeostasis and regulating the levels of DELLA, a growth inhibitor in the GA signaling pathway [7]. GA homeostasis in plants is maintained through coordinating the expression levels of the GA biosynthesis genes, such as GA3OXs and GA200Xs, and the catabolic enzyme genes GA2OXs, thereby regulating floral transition [7,32]. In this study, homologs of GA2OX1 (Unigene0030732) and GA2OX8 (Unigene0073113) were both upregulated in LD10-vs.-SD10 (Fig. 5c; Additional file 14: Table S8). GA2OXs can catalyze the $2 \beta$-hydroxylation of bioactive GAs (such as $\mathrm{GA}_{1}$, $\mathrm{GA}_{3}, \mathrm{GA}_{4}$, and $\mathrm{GA}_{9}$ ), resulting in decreased levels of bioactive Gas [33]. This may be one of the reasons for low $\mathrm{GA}_{3}$ content in shoot apexes of L. gratissima. The main components of GA signaling include the GA receptor GID1B and the growth inhibitors, DELLAs [7]. When GA concentrations increase, the DELLA protein forms a GA-GID1B-DELLA complex that undergoes degradation by the ubiquitination pathway, thereby activating the expression of downstream genes [7]. The GA signaling pathway mainly promotes floral transition by inducing the expression of $S O C 1$ and $L F Y[7,34,35]$. In this study, RGL3 (Unigene0071862) encoding DELLA had low expression in SD10, SD13, and SD19 (Fig. 5d; Additional file 14: Table S8). In contrast, SOC1 (Unigene0039572 and Unigene0039575) and LFY (Unigene0030979) were highly expressed in SD10, SD13, and SD19 (Fig. 5g, h; Additional file 14: Table S8). This showed that low levels of the DELLA protein $\mathrm{RGL}_{3}$ could induce the expression of $S O C 1$ and $L F Y$. Additionally, the GA receptor genes GID1Bs (Unigene0032780, Unigene0032781, and Unigene0063035) were upregulated in LD10-vs.-SD10 (Fig. 5d; Additional file 14: Table S8), further demonstrating that GA promotes floral transition in L. gratissima. However, it may not be $\mathrm{GA}_{1}, \mathrm{GA}_{3}, \mathrm{GA}_{4}$, or $\mathrm{GA}_{9}$ but other active $\mathrm{GAs}$ that took effect. Previous studies indicated that GA has a promoting effect in floral transition in $A$. thaliana $[7,9]$, whereas GA was found to negatively regulate floral transition in woody plants [8]. GA regulation of floral transition in L. gratissima (a woody plant) is similar to herbaceous plants but not woody plants. This unique regulation pattern may be affected by many endogenous and environmental factors, which needs to be further studied in the future.

Other hormones also have some effects in regulating floral transition in L. gratissima. ABA is usually considered a stress-related hormone, but it also plays an important role in plant development [36]. However, there is still debate over the role of $A B A$ in floral transition because both promoting and inhibitory effects were reported [16, 37]. In this study, the ABA synthase gene NCED1 (Unigene0042754 and Unigene0042755) and the catabolic gene CYP707A1 (Unigene0034382) were both upregulated in LD10-vs.-SD10 (Fig. 5c; Additional file 14: Table S8), and the ABA content in the SAMs was maintained at high levels that initially increased from SD0 to SD13 and subsequently declined, reaching its peak on SD13 (Fig. 3). ABF2 is a bZIP transcription factor that binds to ABA. It is also an important component of the glucose signaling pathway [38]. In this study, ABF2 (Unigene0046988) was highly expressed in SD10, and likely participated in floral transition in L. gratissima by mediating the $A B A$ and glucose signaling pathways. In the ABA core signaling pathway, the protein phosphatase PP2C (ABI1, ABI2, HAB1, and 
PP2CA/AHG3) acts as a key negative regulatory factor, which has important regulatory effects on the activation of ABA signaling [39]. When ABA levels increase in plants, the ABA receptors PYR1/PYLs/RCARs bind and inhibit the phosphatase activity of PP2C, thereby activating the ABA signaling pathway [39]. In this study, PYL4 expression was high in SD13, whereas PP2C expression peaked on SD10 but was also high on SD13 (Fig. 5d; Additional file 14: Table S8), suggesting that the activation of the ABA signaling pathway mainly occurred on SD13 and that ABA promoted flower development in L. gratissima through the core signaling pathway. EDL3 is a positive regulator of the ABA signal cascade reactions, and it positively regulates the expression of the central component CONSTANS $(\mathrm{CO})$ in the photoperiod pathway to regulate floral transition [40]. In this study, the expression of EDL3 and COL 12 in the photoperiodic pathway peaked on SD10 (Fig. 5a, d; Additional file 14: Table S8), showing that ABA promoted floral transition in L. gratissima by interacting with EDL3 to induce COL 12 expression.

Plant growth depends on the continuous function of meristems, and CKs have positive effects on SAMs. In this study, the cytokinin synthase gene LOGs and the zeatin 0-glucosyltransferase gene ZOG1 were mainly upregulated in LD10-vs.-SD10 and LD13-vs.-SD13 (Fig. 5c, d; Additional file 14: Table S8). It is known that zeatin O-glucoside plays important roles in the transport and storage of CKs [41]. On the other hand, the trans-zeatin synthase gene CYP735A1 and the cytokinin oxidase/dehydrogenase gene $C K X 7$ were downregulated in LD19-vs.-SD19 (Fig. 5c, d; Additional file 14: Table S8). Zeatin promotes cell division and has an important role in the early stages of flower bud development and cell division. This is a likely the reason zeatin content gradually decreased from SD0 to SD19 (Fig. 3). The CK signaling pathway mainly cross talks with AGAMOUS (AG) to regulate SAM differentiation and maintenance [42]. RPN12A participates in ATP-dependent ubiquitinated protein degradation, which may inhibit the degradation of one or more factors in $\mathrm{CK}$ signaling and balance the proliferation rate of cells during bud development [43]. In this study, AHPS, which are key components in the cytokinin two-component signaling system [44], were highly expressed mainly at SD10, SD13, and SD19; ARR6, which is a CK responsive regulator [44], was significantly upregulated in LD19-vs.-SD19, and RPN12A was upregulated in LD13-vs.-SD13; moreover, AGL8 was highly expressed in SD10, SD13, and SD19 (Fig. 5d, h; Additional file 14: Table S8), demonstrating that CK promotes floral transition and flower development in $L$. gratissima indirectly through the effects of AGL8.

In the JA signaling pathway, JAZ (jasmonate-ZIM domain, TIFY family) and MYC2/3/4 regulate floral transition in plants [7]. In this study, TIFYS and MYC4 were upregulated in LD10-vs.-SD10 (Fig. 5d; Additional file 14: Table S8), showing that the JA signaling pathway promotes floral transition in $L$. gratissima. In SL signaling pathway, D14 negatively regulates SL signals as an SL receptor [45]. In this study, D14 (Unigene0028658) expression was high at the early stage of SD treatment, and as treatment duration increased, its expression level decreased (Fig. 5d; Additional file 14: Table S8), which may have been caused by negative feedback regulation of SL signals by D14, thereby regulating SL changes during floral transition in L. gratissima. CCD7 is a key enzyme in SL biosynthesis [7]. Compared with the LD treatment, $C C D 7$ (Unigene0069349) expression was lower in response to SD treatment and was significantly downregulated in LD10-vs.-SD10 (Fig. 5c; Additional file 14: Table S8), suggesting that SL may inhibit floral transition in L. gratissima. In contrast to the results of this study, recent studies have 
shown that SL inhibits melatonin synthesis, thereby inducing floral transition in $A$. thaliana in an FLCdependent manner [15]. As L. gratissima is a perennial woody plant, there may be differences in SL regulatory mechanisms in floral transition compared with $A$. thaliana, which requires further in-depth studies.

YUC-mediated auxin biosynthesis is vital for the formation of floral organs [46]. In this study, YUC4 was upregulated in LD19-vs.-SD19 (Fig. 5c; Additional file 14: Table S8), whereas IAA content was lowest in SD10 and continuously decreased from SD13 to SD19 (Fig. 3), whereas the auxin response gene SAUR7 was upregulated in LD13-vs.-SD13 and LD19-vs.-SD19. These results showed that auxin does not participate in regulating floral transition in L. gratissima but instead has positive effects on the formation of floral organs.

These hormones interacted with other flowering regulation pathways to further ensure that L. gratissima rapidly responded to changes in environmental and endogenous signals to precisely regulate flowering time.

\section{Flowering pathways during floral transition in L. gratissima}

The photoperiod pathway is involved in plant response to changes to day length and circadian rhythm, making it one of the most important flowering regulation pathways. In the photoperiod pathways of many plants, the bZIP transcription factor FD forms a transient complex in SAMs with the FT protein from leaves to induce the expression of floral meristem identity genes, thereby promoting floral transition [47]. In this study, FD, AP1, FUL, and AGL8 were highly expressed in SD10 and SD13 (Fig. 5a, h), demonstrating that the FD protein directly or indirectly induced the expressions of $A P 1, F U L$, and $A G L 8$, thereby promoting floral transition in L. gratissima. $\mathrm{CO}$ is an important regulatory factor in the photoperiod pathway, and the expression of $\mathrm{CO}$ is regulated by a photoreceptor and circadian rhythm in A. thaliana, and when the expression rhythm of $\mathrm{CO}$ is consistent with the external photoperiod, expression of the downstream gene SOC1 is activated [48]. In this study, CO-like 12 (COL12) was upregulated in LD13-vs.-SD13 (Fig. 5a; Additional file 14: Table S8), showing that the effects of COL12 in flower development in L. gratissima were similar to those of CO in A. thaliana.

The transcription factor LUX is one of the components of evening complex (EC) complex in circadian rhythm, and forms the HOS15-EC-HDA9 histone-modifying complex in A. thaliana to inhibit $G I$ transcription, thereby inhibiting photoperiod-dependent flowering [49]. In this study, LUX was downregulated in LD10-vs.-SD10 (Fig. 5a; Additional file 14: Table S8), indicating that LUX had inhibitory effects on floral transition in L. gratissima. PRR7 positively regulates $C O$ expression to promote floral transition in long-day plants, whereas the $P R R 7 / P R R 3$ genes delay floral transition by inhibiting $C O$ expression in short-day plants [50]. In this study, PRR7 was downregulated in LD13-vs.-SD13 (Fig. 5a; Additional file 14: Table S8), showing that PRR7 inhibits floral transition in L. gratissima, which was similar to the other short-day plants. In $A$. thaliana, FKF1 could degrade CDF1 (factor inhibiting CO transcription) to regulate $C O$ expression and could directly bind to $\mathrm{CO}$, or inhibit $C O P 1$ to stabilize $C O$ expression, thereby promoting flowering [51]. However, FKF1 was downregulated in LD13-vs.-SD13 (Fig. 
5a; Additional file 14: Table S8), which was not consistent with COL 12 expression. This indicated that FKF1 inhibited floral transition in L. gratissima and does not interact with COL12, but other mechanisms may be present that require further study. NF-Ys interacts with $\mathrm{CO}$ in the photoperiod pathway to directly regulate SOC1 transcription [34]. In this study, NF-YS, COL 12, and SOC1 were highly expressed in SD10 and SD19 (Fig. 5a, h), showing that NF-Ys may interact with COL12 in the photoperiod pathway in $L$. gratissima to induce $\mathrm{SOC} 1$ expression, thereby positively regulating floral transition and flowering development in L. gratissima.

Previous studies showed that ambient temperature-associated EARLI1 regulated critical genes in the LD photoperiod pathway in $A$. thaliana to promote FLC expression and delayed flowering time [52]. In contrast, $p E A R L / 1$ was upregulated in LD13-vs.-SD13 and LD19-vs.-SD19 in this study (Fig. 5b; Additional file 14: Table S8), indicating that $p E A R L / 1$ promoted floral transition and flower development in $L$. gratissima.

In $A$. thaliana, age signals negatively regulate miR156 levels to promote $S P L$ accumulation [20]. At SAMs, SPLs target FUL and $S O C 1$ or directly regulate $A P 1$ transcription to promote flowering [53]. In this study, SPL4 was upregulated in LD10-vs.-SD10, LD13-vs.-SD13, and LD19-vs.-SD19 (Fig. 5f; Additional file 14: Table S8), which was consistent with the expression patterns of $S O C 1, F U L$, and $A P 1$ (Fig. $5 \mathrm{~g}, \mathrm{~h}$ ), indicating that the aging pathway promoted floral transition and flower development in L. gratissima through SPL4-induced expression of FUL, SOC1, and AP1.

The floral integrators SOC1 and AGL24 integrate various flowering signals from photoperiod, temperature, hormone, and age-related signals to activate or inhibit downstream floral meristem identity genes, and ultimately lead to the transformation of vegetative to floral meristems in plants [5]. SOC1 can be indirectly activated by $\mathrm{CO}$ [54]. At SAMs, when $\mathrm{SOC1}$ is activated, SOC1 and AGL24 form a heterodimer to directly activate $L F Y$ [55]. In this study, SOC1, AGL24, and $L F Y$ were highly expressed in SD10, showing that SOC1 and AGL24 can jointly promote $L F Y$ at this period to promote floral transition in $L$. gratissima. During early flower development, AP1 activates A function to inhibit SOC1 and AGL24 expression to prevent flowering reversion [54]. In SD19, AGL24 and SOC1 expression decreased and AP1 expression increased (Fig. $5 \mathrm{~g}, \mathrm{~h}$ ). These changes may prevent differentiated floral meristems from undergoing flowering reversion.

SEPs are important regulatory factors during flower development and form a heterodimer with AP1 to regulate genes during floral meristem development [56]. In this study, SEPS were highly expressed in SD10, SD13, and SD9, which was consistent with AP1 expression (Fig. 5h), showing that AP1 mediated positive regulation of floral transition and early flower development in L. gratissima by SEPs. In Arabidopsis, SVP is a flowering inhibitor and plays a role in floral transition by directly inhibiting SOC1 expression at SAMs and leaves [57]. In this study, SVP had low expressions in SD10, SD13, and SD19, whereas SOC1 expression was high (Fig. 5g, h). indicating that low levels of SVP induced SOC1 expression to promote floral transition and flower formation in L. gratissima. 
TFL1 is a key regulatory factor of floral transition and inflorescence meristem development in $A$. thaliana. TFL1 and FT have highly conserved amino acid sequences but opposite gene functions: FT promotes flowering, whereas TFL1 inhibits flowering [58]. Previous studies showed that TFL1 negatively regulated transcription of the target gene $F D$, thereby regulating the flowering time and inflorescence meristem development [59]. In this study, TFL 1 had low expression at SD10 and SD13, which is the opposite of FD expression (Fig. 5a, h), indicating that low levels of TFL1 promoted FD expression and, therefore, floral transition in L. gratissima.

Fig. 6 shows the hypothetical model of the regulatory network of SD photoperiod-induced floral transition in L. gratissima, involved in the regulation of multiple flowering signals in floral transition, including signals for photoperiod, phytohormones (GA, ABA, CK, JA, and SL), sugar, ambient temperature, age, and floral integrator and floral meristem identity genes.

\section{Conclusions}

Our study enables a comprehensive understanding of the gene expression patterns occurring during SD photoperiod-induced floral transition in L. gratissima. The histological, endogenous substance contents, and differential gene expression analyzes showed that short-day photoperiod activated systemic responses in L. gratissima and induced the generation of flowering signals in the photoperiod pathway. Furthermore, a complex regulatory network, including GA, ABA, CK, JA, and SL signals, sugar signals, and temperature and age signals was formed through the integration of SOC1 and AGL24. The outcomes of this study will aid in understanding flowering time regulation in L. gratissima at the molecular level, provide theoretical guidance for achieving year-round production, and further provide a reference for understanding the regulatory mechanisms of flowering time in other woody plants.

\section{Methods}

\section{Plant materials, growth conditions, and light treatments}

L. gratissima cultivar 'Xiangfei' cuttings were obtained from the central Yunnan Plateau experimental station of Research Institute of Resources Insects, Chinese Academy of Forestry (Yunnan, China; $25^{\circ} 13^{\prime} \mathrm{N}$, $102^{\circ} 12^{\prime} \mathrm{E}, 1826 \mathrm{~m}$ a.s.I.). In mid-December 2016, cuttings with two stem nodes and shoot apexes were planted in a mixed matrix (peat and perlite at a $3: 1$ ratio) and grown in an $18-25^{\circ} \mathrm{C}$ greenhouse under natural lighting. Cuttings with roots were transplanted into pots and maintained in the same greenhouse under natural lighting. To prevent these plants from being induced by SD photoperiod, shoot apical meristems (SAM) were removed from all plants when 2-3 new stem nodes were formed, and highpressure sodium lamps were used for additional lighting during 22:00-02:00 (night-break treatment) (Fig. 1c). In addition, considering the effects of individual developmental age on flowering time [60], some plants were placed in the natural environment as controls and the time when flower bud differentiation occurred in these plants was used as the start time for photoperiod treatments. On 10 August 2017 (when flower buds began to appear in some natural control plants), plants with the same number of branches 
longer than $5 \mathrm{~cm}$ were selected from among the night-break treatment plants, and were then subjected to either LD (night-break treatment as described above) or to SD (10 h light/14 h dark) (Fig. 1d) for a further 90 days. Shoot apexes of the main branches of SD and LD plants were sampled during 09:00-11:30 every 3-5d after the initiation of the photoperiod treatments. For each stage, $10-20$ shoot apexes were packed together into each of the 10 biological replicates, of which one biological replicate was rapidly immersed into FAA fixative (50\% ethanol: acetic acid: formaldehyde, 18:1:1) for morphological analysis, whereas the remaining nine biological replicates were snap-frozen in liquid nitrogen and then stored at $80^{\circ} \mathrm{C}$ for measurements of soluble sugar and endogenous hormone contents, as well as RNA extraction.

\section{Morphological anatomical observations}

Ten FAA-fixed shoot apexes of SD and LD plants at each stage were made into sections with a thickness of 8-10 $\mu \mathrm{m}$ using paraffin section method [61], and were stained with safranin 0-fast green, and then were mounted with neutral resin. Finally, the process of bud development was observed under a Carl Zeiss Axio Scope A1 Microscope (Carl Zeiss Microscopy GmbH, Göttingen, Germany).

\section{Measurements of soluble sugar and endogenous hormone contents}

According to the anatomical observation results, samples from the SD treatment at five stages (0 d [SD0], $7 \mathrm{~d}$ [SD7], $10 \mathrm{~d}$ [SD10], $13 \mathrm{~d}$ [SD13], and $19 \mathrm{~d}$ [SD19]) close to flower bud differentiation (Fig. 2) were selected for measurements of soluble sugar and endogenous hormone contents of three biological replicates. For each of the three biological replicates from each stage, soluble sugar contents were measured using sulfuric acid-anthrone colorimetric assays [62], and endogenous hormones $\left(\mathrm{GA}_{3}\right.$, indoleacetic acid [IAA], ABA, and zeatin [ZT]) were quantified with high-performance liquid chromatography-mass spectrometry (HPLC-MS; Aglient1290, Nanjing, China; AB 6500, Nanjing, China) [63]. Before comparing changes in the soluble sugar and hormone contents among the five stages, the Shapiro-Wilk test and Levene test were used to analyze the normality and homogeneity of variance of each dataset. Because the four sets of data did not follow a normal distribution $(p<0.05)$, a KruskalWallis $\mathrm{H}$ test was employed for analysis of significant differences, and false discovery rate (FDR) was used for the multiple testing correction of significant $p$-values. Additionally, the Tukey-Kramer method was used for post-hoc testing of soluble sugar and hormone contents at the five stages. The above analyzes were performed in the "car" and "stats" packages in R software and the data were expressed as the mean \pm SD.

\section{Transcriptome sequencing and data analysis}

Likewise, based on the anatomical observation results, samples from the SD and LD treatments at the four stages (7 d [SD7 or LD7], $10 \mathrm{~d}$ [SD10 or LD10], $13 \mathrm{~d}$ [SD13 or LD13], and $19 \mathrm{~d}$ [SD19 or LD19]) close to flower bud differentiation of SD plants (Fig. 2) were selected for RNA extraction. Total RNA extracted from each of the three biological replicates was divided into two parts, of which one was used for RNAseq and the other was used for quantitative real-time PCR (qRT-PCR) validation. RNA was extracted with the Plant total RNA Kit (Tiangen, Beijing, China) following manufacturer's instructions. The cDNA library 
construction and paired-end sequencing were conducted with an Illumina HiSeq ${ }^{\mathrm{TM}} 4000$ (Illumina, San Diego, California, USA) at the Gene Denovo Biotechnology Company (Guangzhou, China). The generated raw reads were filtered by removing adapter sequences and ambiguous reads $(\mathrm{N}>10 \%)$ and low-quality reads (more than $40 \%$ of bases with $Q$ value $\leq 20$ ) to obtain high-quality clean reads. Finally, the Trinity software [64] was used for de novo assembly of filtered clean reads to obtain unigenes.

All non-redundant unigenes were aligned with selected cut-offs of $E$-value $\leq 1 \mathrm{e}^{-5}$ to six protein databases, including the NR (the NCBI non-redundant protein databases), KOG (EuKaryotic Orthologous Groups), KEGG (Kyoto Encyclopedia of Genes and Genomes), Swiss-Prot, eggNOG (evolutionary genealogy of genes: Non-supervised Orthologous Groups), and Pfam (Protein families database of alignments and hidden Markov models). Based on the NR annotation results, these unigenes were also annotated for GO (Gene Ontology) using the Blast2GO software [65], and then GO functional classification of unigenes was obtained by WEGO software [66].

\section{Quantitative real-time PCR analysis}

Quantitative real-time PCR (qRT-PCR) was conducted on nine flowering-related unigenes in this study, including COP1 (Unigene0031506), ZTL (Unigene0041339), FKF1 (Unigene0038380), GI (Unigene0051409), ELF3 (Unigene0051761), PRR1 (Unigene0045946), PRR7 (Unigene0003564), PRR5 (Unigene0047475), and $L H Y$ (Unigene0035686). To accurately measure gene expression levels, the ACT7/EF1- $a$ combination obtained from the past screening was used as an internal reference gene for standardization and correction (Additional file 16: Supplementary Data). Primer3 software [67] was used to design specific primers for each gene (Additional file 17: Table S9). The KR106 FastQuantity RT Kit (with gDNase) (Tiangen, Beijing, China) was used for reverse transcription of $1 \mu \mathrm{g}$ total RNA into cDNA according to the manufacturer's instructions. The StepOnePlus ${ }^{\text {TM }}$ Real-Time PCR System (Thermo Scientific, Wilmington, DE, USA) was used for qRT-PCR in a $20 \mu \mathrm{L}$ reaction system, including $4 \mu \mathrm{L}$ of $50 \mathrm{ng}$ cDNA template, $10 \mu \mathrm{L}$ of $2 \times$ qPCR Master Mix (Tiangen, Beijing, China), $0.4 \mu \mathrm{L}$ each of $10 \mu \mathrm{M}$ forward

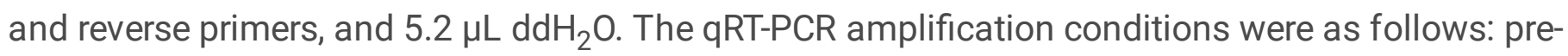
denaturation at $95^{\circ} \mathrm{C}$ for $90 \mathrm{~s}$, followed by 40 cycles of denaturation at $95^{\circ} \mathrm{C}$ for $5 \mathrm{~s}$, annealing at $60^{\circ} \mathrm{C}$ for $15 \mathrm{~s}$, extension at $72^{\circ} \mathrm{C}$ for $20 \mathrm{~s}$, followed by a final extension step at $72^{\circ} \mathrm{C}$ for $5 \mathrm{~min}$, After amplification, a $65-95^{\circ} \mathrm{C}$ melting curve analysis was conducted to measure product specificity. The $2^{-\Delta \Delta C t}$ method [68] was used to calculate the relative expression levels of the genes in the qRT-PCR experiment.

\section{Identification and functional enrichment of differentially expressed genes (DEGs)}

The Reads Per kb per Million reads (RPKM) method was used to evaluate unigene expression levels [69]. EdgeR package version 3.8 [70] was used for differential expression analysis of multiple comparisons, and the FDR method [71] was used for the correction of $P$-values. FDR $<0.05$ and $\mid \log _{2}$ Ratio| $>1$ were used as criteria to screen for DEGs between various groups (ratio refers to the RPKM ratios of SD7/LD7, SD10/LD10, SD13/LD13, and SD19/LD19). 
The Mercator online tool (https://www.plabipd.de/portal/mercator-sequence-annotation) was employed for gene function predictions for the DEGs with a BLAST-CUTOFF of 50. The obtained mapping files were uploaded to MapMan version 3.6 [72] for the functional analysis of DEGs. Wilcoxon rank-sum test was used to analyze the $\log _{2}$ Ratio of DEGs in each comparison before MapMan version 3.6 [72] was used for visualization of the results.

\section{Co-expression network analysis}

Weighted gene co-expression network analysis (WGCNA) [13] was employed to generate the coexpression network modules of DEGs. The parameter settings used were soft threshold $=20$, minModuleSize $=30$, TOMType $=$ signed, mergeCutHeight $=0.25$, and default values were used for the remaining parameters. The eigengene value of every module was calculated and the associations between every gene in eight samples were tested. KOBAS 3.0 [73] was used for GO enrichment analysis of genes in the clustering modules. Cytoscape version 3.7.1 [74] was used for visualization of the coexpression network.

\section{List Of Abbreviations}

ABA: abscisic acid; AG: AGAMOUS; AGL24: AGAMOUSLIKE24; AP1: APETALA1; BGLU24: BETAGLUCOSIDASE 24; CAB40: CHLOROPHYLL A-B BINDING PROTEIN; CCD7: CAROTENOID CLEAVAGE DIOXYGENASE 7; CK: cytokinin; CO: CONSTANS; COL12: CONSTANS-LIKE 12; CYP734A1: CYTOCHROME P450 734A1; D14: DWARF14; DEG: differentially expressed gene; EC: evening complex; EDL3: EID1-LIKE F-BOX PROTEIN 3; FKF1: FLAVIN-BINDING KELCH REPEAT F-BOX PROTEIN 1; FT: FLOWERING LOCUS T; FUL: FRUITFULL; GA: gibberellin; GA2OX1: GIBBERELLIN 2-BETA-DIOXYGENASE 1; GOLS2: GALACTINOL SYNTHASE 2; HK: HEXOKINASE; HPLC-MS: high performance liquid chromatography-mass spectrometry; HSFs: HEAT STRESS TRANSCRIPTION FACTORS; HSPS: HEAT SHOCK PROTEIN/ COGNATE; IAA: indole3-acetic acid; JA: jasmonic acid; JMT: JASMONATE O-METHYLTRANSFERASE; LD: Iong day; LFY: LEAFY; NF-Y: nuclear factor Y; PRR7: PSEUDO-RESPONSE REGULATOR 7; RFS: RAFFINOSE SYNTHASE; SD: short day; SEP3: SEPALLATA3; SEPs: SEPALLATAs; SL: strigolactone; SOC1: SUPPRESSOR OF OVEREXPRESSION OF CONSTANS 1; SPL4: SQUAMOSA PROMOTER-BINDING-LIKE PROTEIN 4; SVP: SHORT VEGETATIVE PHASE; T6P: trehalose-6-phosphate; TFL1: TERMINAL FLOWER 1; TPS: TREHALOSE-6-PHOSPHATE SYNTHSE; UGT74F1: UDP-GLYCOSYLTRANSFERASE 74F1; WGCNA: weighted gene co-expression network analysis; ZT: zeatin

\section{Declarations}

\section{Acknowledgments}

The authors would like to thank Guangzhou Genedenovo Biotechnology Co., Ltd for assisting in RNA sequencing. 
ZHL, YMW, and HM conceived and designed the study. XFL, YMW, JA, XJZ, YRC, and XXL conducted the experiment and collected the plant materials. XFL and YMW analyzed and interpreted the data. XFL and YMW wrote the manuscript. YMW, ZHL, and HM revised the manuscript. All authors read and approved the final version of the manuscript.

\section{Funding}

This work was funded by Key Research and Development Program of Yunnan Province (2018BB012 to Z.-H.L.), the Fundamental Research Funds for the Central Non-profit Research Institution of CAF (CAFYBB2017MB014 to Y.-M.W.), the Fundamental Research Funds for the Central Non-profit Research Institution of CAF (CAFYBB2019ZB007 to Z.-H.L.), and Ten Thousand Talent Program of Yunnan Province (grant to H.M.).

\section{Availability of data and materials}

The raw data of RNA sequencing from this study have been deposited into the NCBI Sequence Read Archive (SRA) database under the accession number SRP273656.

\section{Ethics approval and consent to participate}

Not applicable.

\section{Consent for publication}

Not applicable.

\section{Competing interests}

The authors declare that they have no competing interests.

\section{References}

1. Cho L, Yoon J, An G. The control of flowering time by environmental factors. Plant J. 2017;90:708-19. doi:10.1111/tpj.13461.

2. Shang J, Tian J, Cheng H, Yan Q, Li L, Jamal A, et al. The chromosome-level wintersweet (Chimonanthus praecox) genome provides insights into floral scent biosynthesis and flowering in winter. Genome Biol. 2020;21:200.

3. Liu L, Zhang Y, Yu H. Florigen trafficking integrates photoperiod and temperature signals in Arabidopsis. J Integr Plant Biol. 2020. doi:10.1111/jipb.13000.

4. Zhang Y, Li Z, Chen N, Huang Y, Huang S. Phase separation of Arabidopsis EMB1579 controls transcription, mRNA splicing, and development. PLoS Biol. 2020;18:e3000782. doi:10.1371/journal.pbio.3000782. 
5. BIÜmel M, Dally N, Jung C. Flowering time regulation in crops - what did we learn from Arabidopsis? Curr Opin Biotech. 2015;32:121-9. doi:10.1016/j.copbio.2014.11.023.

6. Khan MRG, Ai X, Zhang J. Genetic regulation of flowering time in annual and perennial plants. Wiley Interdiscip Rev RNA. 2014;5:347-59. doi:10.1046/j.1365-313X.2003.01862.x.

7. Bao S, Hua C, Shen L, Yu H. New insights into gibberellin signaling in regulating flowering in Arabidopsis. J Integr Plant Biol. 2020;62:118-31. doi:10.1111/jipb.12892.

8. Li J, Pan B, Niu L, Chen M, Tang M, Xu Z. Gibberellin inhibits floral initiation in the perennial woody plant Jatropha curcas. J Plant Growth Regul. 2018;37:999-1006. doi:.1007/s00344-018-9797-8.

9. Yamaguchi N, Winter CM, Wu M, Kanno Y, Yamaguchi A, Seo M, et al. Gibberellin acts positively then negatively to control onset of flower formation in Arabidopsis. Science. 2014;344:638-41. doi:10.1126/science.1250498.

10. Zhou W, Li D, Wang, H. A set of novel microsatellite markers developed for a distylous species Luculia gratissima (Rubiaceae). Int J Mol Sci. 2011;12:6743-8. doi:10.3390/ijms12106743.

11. Wan Y, Ma H, Zhao Z, Li T, Liu X, Liu X, et al. Flowering response and anatomical study on process of flower bud differentiation for Luculia gratissima 'Xiangfei' under different photoperiods. Acta Bot Boreal-Occident Sin. 2018;38:1659-66. Chinese.

12. Ramšak Ž, Baebler Š, Rotter A, Korbar M, Mozetič I, Usadel B, et al. GoMapMan: integration, consolidation and visualization of plant gene annotations within the MapMan ontology. Nucleic Acids Res. 2014 Jan 1;42(D1):D1167-75. doi:10.1093/nar/gkt1056.

13. Langfelder P, Horvath S. WGCNA: an R package for weighted correlation network analysis. BMC Bioinformatics. 2008;9:559. doi:10.1186/1471-2105-9-559.

14. Lin T, Walworth A, Zong $\mathrm{X}$, Danial GH, Tomaszewski EM, Callow $\mathrm{P}$, et al. VcRR2 regulates chillingmediated flowering through expression of hormone genes in a transgenic blueberry mutant. Hortic Res. 2019;6:96. doi:10.1038/s41438-019-0180-0.

15. Zhang Z, Hu Q, Liu Y, Cheng P, Cheng H, Liu W, et al. Strigolactone represses the synthesis of melatonin, thereby inducing floral transition in Arabidopsis thaliana in an FLC-dependent manner. J Pineal Res. 2019;67:e12582. doi:10.1111/jpi.12582.

16. Shu K, Luo X, Meng Y, Yang W. Toward a molecular understanding of abscisic acid actions in floral transition. Plant Cell Physiol. 2018;59:215-21. doi:10.1093/pcp/pcy007.

17. Nelson RJ, Denlinger DL, Somers DE, editors. Photoperiodism: the biological calendar. England: Oxford University Press; 2010 Jan 27.

18. Dong $X$, Yan Y, Jiang B, Shi Y, Jia Y, Cheng J, et al. The cold response regulator CBF1 promotes Arabidopsis hypocotyl growth at ambient temperatures. EMBO J. 2020;39:103630. doi:10.15252/embj.2019103630.

19. Meng X, Li Y, Yuan Y, Zhang Y, Li H, Zhao J, et al. The regulatory pathways of distinct flowering characteristics in Chinese jujube. Hortic Res. 2020;7:123. doi:10.1038/s41438-020-00344-7. 
20. Yao T, Park BS, Mao H, Seo JS, Ohama N, Li Y, et al. Regulation of flowering time by SPL10/MED25 module in Arabidopsis. New Phytol. 2019;224:493-504. doi:10.1111/nph.15954.

21. Gregis V, Sessa A, Colombo L, Kater MM. AGL24, SHORT VEGETATIVE PHASE, and APETALA1 redundantly control AGAMOUS during early stages of flower development in Arabidopsis. Plant Cell. 2006;18:1373-82. doi:10.1111/j.1365-313X.2008.03648.x.

22. Chang G, Yang W, Zhang Q, Huang J, Yang Y, Hu X. ABI5-BINDING PROTEIN2 coordinates CONSTANS to delay flowering by recruiting the transcriptional corepressor TPR2. Plant Physiol. 2019;179:477-90. doi:10.1104/pp.18.00865.

23. Lebon G, Wojnarowiez G, Holzapfel B, Fontaine F, Vaillant-Gaveau N, Clement C. Sugars and flowering in the grapevine (Vitis vinifera L.). J Exp Bot. 2008;59:2565-78. doi:10.1093/jxb/ern135.

24. Ortiz-Marchena MI, Romero JM, Valverde F. Photoperiodic control of sugar release during the floral transition: What is the role of sugars in the florigenic signal? Plant Signal Behav. 2015;10:e1017168. doi:10.1080/15592324.2015.1017168.

25. Bernier G, Périlleux C. A physiological overview of the genetics of flowering time control. Plant Biotechnol J. 2005;3:3-16. doi:10.1111/j.1467-7652.2004.00114.x.

26. Smeekens S, Ma J, Hanson J, Rolland F. Sugar signals and molecular networks controlling plant growth. Curr Opin Plant Biol. 2010;13:273-8. doi:10.1016/j.pbi.2009.12.002.

27. Abelenda JA, Bergonzi S, Oortwijn M, Sonnewald S, Du M, Visser RGF, et al. Source-sink regulation is mediated by interaction of an FT homolog with a SWEET protein in potato. Curr Biol. 2019;29:1-9. doi:10.1016/j.cub.2019.02.018.

28. Kataya ARA, Elshobaky A, Heidari B, Dugassa N, Thelen JJ, Lillo C. Multi-targeted trehalose-6phosphate phosphatase I harbors a novel peroxisomal targeting signal 1 and is essential for flowering and development. Planta. 2020;251:98. doi:10.1007/s00425-020-03389-z.

29. Wahl V, Ponnu J, Schlereth A, Arrivault S, Langenecker T, Franke A, et al. Regulation of flowering by trehalose-6-phosphate signaling in Arabidopsis thaliana. Science. 2013;339:704-7. doi:10.1126/science.1230406.

30. Teng S, Rognoni S, Bentsink L, Smeekens S. The Arabidopsis GSQ5/DOG1 Cvi allele is induced by the ABA-mediated sugar signaling pathway, and enhances sugar sensitivity by stimulating $A B / 4$ expression. Plant J. 2008;55:372-81. doi:10.1111/j.1365-313X.2008.03515.x.

31. Moore B, Zhou L, Rolland F, Hall Q, Cheng W, Liu Y, et al. Role of the Arabidopsis glucose sensor HXK1 in nutrient, light, and hormonal signaling. Science. 2003;300:332-6. doi:10.1126/science.1080585.

32. Mateos JL, Madrigal P, Tsuda K, Rawat V, Richter R, Romera-Branchat M, et al. Combinatorial activities of SHORT VEGETATIVE PHASE and FLOWERING LOCUS C define distinct modes of flowering regulation in Arabidopsis. Genome Biol. 2015;16:31. doi:10.1186/s13059-015-0597-1.

33. Rieu I, Eriksson S, Powers SJ, Gong F, Griffiths J, Woolley L, et al. Genetic analysis reveals that $\mathrm{C}_{19}-\mathrm{GA}$ 2-oxidation is a major gibberellin inactivation pathway in Arabidopsis. Plant Cell. 2008;20:2420-36. doi:10.1105/tpc.108.058818. 
34. Hou X, Zhou J, Liu C, Liu L, Shen L, Yu H. Nuclear factor Y-mediated H3K27me3 demethylation of the SOC1 locus orchestrates flowering responses of Arabidopsis. Nat Commun. 2014;5:4601. doi:10.1038/ncomms5601.

35. Blázquez MA, Green R., Nilsson O, Sussman MR, Weigel D. Gibberellins promote flowering of Arabidopsis by activating the LEAFY promoter. Plant Cell. 1998;10:791-800. doi:1010.1105/tpc.10.5.791.

36. Yoshida T, Christmann A, Yamaguchi-Shinozaki K, Grill E, Fernie AR. Revisiting the basal role of ABA - roles outside of stress. Trends Plant Sci. 2020;24:625-35. doi:10.1016/j.tplants.2019.04.008.

37. Xiong F, Ren JJ, Yu Q, Wang YY, Lu CC, Kong LJ, et al. AtU2AF65b functions in abscisic acid mediated flowering via regulating the precursor messenger RNA splicing of $A B I 5$ and FLC in Arabidopsis. New Phytol. 2019;223:277-92. doi:10.1111/nph.15756.

38. Kim S, Kang J, Cho D, Park JH, Kim SY. ABF2, an ABRE-binding bZIP factor, is an essential component of glucose signaling and its overexpression affects multiple stress tolerance. Plant $\mathrm{J}$. 2004;40:75-87. doi:10.1111/j.1365-313X.2004.02192.x.

39. Tischer SV, Wunschel C, Papacek M, Kleigrewe K, Hofmann T, Christmann A, et al. Combinatorial interaction network of abscisic acid receptors and coreceptors from Arabidopsis thaliana. PNAS. 2017;114:10280-5. doi:10.1073/pnas.1706593114.

40. Koops P, Pelser S, Ignatz M, Klose C, Marrocco-Selden K, Kretsch T. EDL3 is an F-box protein involved in the regulation of abscisic acid signaling in Arabidopsis thaliana. J Exp Bot. 2011;62:5547-60. doi:10.1093/jxb/err236.

41. Kiran NS, Benková E, Reková A, Dubová J, Malbeck J, Palme K, et al. Retargeting a maize $\beta$ glucosidase to the vacuole - Evidence from intact plants that zeatin-O-glucoside is stored in the vacuole. Phytochemistry. 2012;79:67-77. doi:10.1016/j.phytochem.2012.03.012.

42. Zhang K, Wang R, Zi H, Li Y, Cao X, Li D, et al. AUXIN RESPONSE FACTOR3 regulates floral meristem determinacy by repressing cytokinin biosynthesis and signaling. Plant Cell. 2018;30:32446. doi:10.1105/tpc.17.00705.

43. Ryu MY, Cho SK, Kim WT. RNAi suppression of RPN12a decreases the expression of type- $A$ ARRs, negative regulators of cytokinin signaling pathway, in Arabidopsis. Mol Cells. 2009;28:375-82. doi:10.1007/s10059-009-0132-x.

44. Liu Z, Yuan L, Song X, Yu X, Sundaresan V. AHP2, AHP3, and AHP5 act downstream of CKI1 in Arabidopsis female gametophyte development. J Exp Bot. 2017;68:3365-73. doi:10.1093/jxb/erx181.

45. Chevalier F, Nieminen K, Sánchez-Ferrero JC, Rodríguez ML, Chagoyen M, Hardtke CS, et al. Strigolactone promotes degradation of DWARF14, an a/ $\beta$ hydrolase essential for strigolactone signaling in Arabidopsis. Plant Cell. 2014;26:1134-50. doi:10.1105/tpc.114.122903.

46. Cheng Y, Dai X, Zhao Y. Auxin biosynthesis by the YUCCA flavin monooxygenases controls the formation of floral organs and vascular tissues in Arabidopsis. Gene Dev. 2006;20:1790-9. doi:10.1101/gad.1415106. 
47. Abe M, Kosaka S, Shibuta M, Nagata K, Uemura T, Nakano A, et al. Transient activity of the florigen complex during the floral transition in Arabidopsis thaliana. Development. 2019;146:dev171504. doi:10.1242/dev.171504.

48. Goretti D, Silvestre M, Collani S, Langenecker T, Méndez C, Madueno F, et al. TERMINAL FLOWER1 functions as a mobile transcriptional cofactor in the shoot apical meristem. Plant Physiol. 2020;182:2081-95. doi:10.1104/pp.19.00867.

49. Park HJ, Baek D, Cha J, Liao X, Kang S, McClung RC, et al. HOS15 interacts with the histone deactetylase HDA9 and the Evening Complex to epigenetically regulate the floral activator GIGANTEA. Plant Cell. 2019;31:37-51. doi:10.1105/tpc.18.00721.

50. Nakamichi N, Kudo T, Makita N, Kiba T, Kinoshita T, Sakakibara H. Flowering time control in rice by introducing Arabidopsis clock-associated PSEUDO-RESPONSE REGULATOR 5. Biosci Biotech Bioch. 2020;84:970-9. doi:10.1080/09168451.2020.1719822.

51. Lee BD, Kim MR, Kang MY, Cha JY, Han SH, Nawkar GM, et al. Author Correction: The F-box protein FKF1 inhibits dimerization of COP1 in the control of photoperiodic flowering. Nat Commun. 2018;9:553. doi:10.1038/s41467-018-02966-x.

52. Shi Y, Zhang X, Xu ZY, Li L, Zhang C, Schläppi M, et al. Influence of EARLI1-like genes on flowering time and lignin synthesis of Arabidopsis thaliana. Plant Biology. 2011;13:731-9. doi:10.1111/j.14388677.2010.00428.x.

53. Wang J, Czech B, Weigel D. miR156-regulated SPL transcription factors define an endogenous flowering pathway in Arabidopsis thaliana. Cell. 2009;138:738-49. doi:10.1016/j.cell.2009.06.014.

54. Lee J, Lee I. Regulation and function of SOC1, a flowering pathway integrator. J Exp Bot. 2010;61:2247-54. doi:10.1093/jxb/erq098.

55. Lee J, Oh M, Park H, Lee I. SOC1 translocated to the nucleus by interaction with AGL24 directly regulates $L E A F Y$. Plant J. 2008;55:832-43. doi:10.1111/j.1365-313X.2008.03552.x.

56. Jetha K, Theißen G, Melzer R. Arabidopsis SEPALLATA proteins differ in cooperative DNA-binding during the formation of floral quartet-like complexes. Nucleic Acids Res. 2014;42:10927-42. doi:10.1093/nar/gku755.

57. Li D, Liu C, Shen L, Wu Y, Chen H, Robertson M, et al. A repressor complex governs the integration of flowering signals in Arabidopsis. Dev Cell. 2008;15:110-20. doi:10.1016/j.devcel.2008.05.002.

58. Jin S, Nasim Z, Susila H, Ahn JH. Evolution and functional diversification of FLOWERING LOCUS T/TERMINAL FLOWER 1 family genes in plants. Semin Cell Dev Biol. 2020. doi:10.1016/j.semcdb.2020.05.007.

59. Hanano S, Goto K. Arabidopsis TERMINAL FLOWER1 is involved in the regulation of flowering time and inflorescence development through transcriptional repression. Plant Cell. 2011;23:3172. doi:10.1105/tpc.111.088641.

60. Evans MR, Wilkins HF, Hackett WP. Meristem ontogenetic age as the controlling factor in long-day floral initiation in poinsettia. J Am Soc Hortic Sci. 1992;117:961-5. doi:10.21273/JASHS.117.6.961. 
61. Fischer AH, Jacobson KA, Rose J, Zeller R. Paraffin embedding tissue samples for sectioning. CSH Protoc. 2008:pdb.prot4989. doi:10.1101/pdb.prot4989.

62. Wang $\mathrm{C}$, Zhang H, Li W, Ye J. Chemical constituents and structural characterization of polysaccharides from four typical bamboo species leaves. Molecules. 2015;20:4162-79. doi:10.3390/molecules20034162.

63. Pan X, Welti R, Wang X. Quantitative analysis of major plant hormones in crude plant extracts by high-performance liquid chromatography-mass spectrometry. Nat Protoc. 2010;5:986-92. doi:10.1038/nprot.2010.37.

64. Grabherr MG, Haas BJ, Yassour M, Levin JZ, Thompson DA, Amit I, et al. Full-length transcriptome assembly from RNA-Seq data without a reference genome. Nat Biotechnol. 2011;29:644-52. doi:10.1038/nbt.1883.

65. Conesa A, Gotz S, Garcia-Gomez JM, Terol J, Talon M, Robles M. Blast2GO: a universal tool for annotation, visualization and analysis in functional genomics research. Bioinformatics. 2005;21:3674-6. doi:10.1093/bioinformatics/bti610.

66. Ye J, Fang L, Zheng H, Zhang Y, Chen J, Zhang Z, et al. WEGO: a web tool for plotting GO annotations. Nucleic Acids Res. 2006;34:W293-7. doi:10.1093/nar/gkl031.

67. Rozen S, Skaletsky H. Primer3 on the WWW for general users and for biologist programmers. Methods Mol Biol. 2000;132:365-86. doi:10.1385/1-59259-192-2:365.

68. Livak KJ, Schmittgen TD. Analysis of relative gene expression data using real-time quantitative PCR and the $2^{-\triangle \Delta C T}$ method. Methods. 2001;25:402-8. doi:10.1006/meth.2001.1262.

69. Mortazavi A, Williams BA, McCue K, Schaeffer L, Wold B. Mapping and quantifying mammalian transcriptomes by RNA-Seq. Nat Methods. 2008;5:621-8. doi:10.1038/nmeth.1226.

70. Robinson MD, Mccarthy DJ, Smyth GK. edgeR: a Bioconductor package for differential expression analysis of digital gene expression data. Bioinformatics. 2010;26:139-40. doi:10.1093/bioinformatics/btp616.

71. Benjamini $Y$, Yekutieli D. The control of the false discovery rate in multiple testing under dependency. Ann Stat. 2001;29:1165-88. doi:10.1214/aos/1013699998.

72. Thimm O, Bläsing O, Gibon Y, Nagel A, Meyer S, Krüger P, et al. MAPMAN: a user-driven tool to display genomics data sets onto diagrams of metabolic pathways and other biological processes. Plant $\mathrm{J}$. 2004;37:914-39. doi:10.1111/j.1365-313x.2004.02016.x.

73. Xie C, Mao X, Huang J, Ding Y, Wu J, Dong S, et al. KOBAS 2.0: a web server for annotation and identification of enriched pathways and diseases. Nucleic Acids Res. 2011;39:W316-22. doi:10.1093/nar/gkr483.

74. Shannon P, Markiel A, Ozier O, Baliga NS, Wang JT, Ramage D, et al. Cytoscape: a software environment for integrated models of biomolecular interaction networks. Genome Res. 2003;13:2498-504. doi:10.1101/gr.1239303.

\section{Figures}




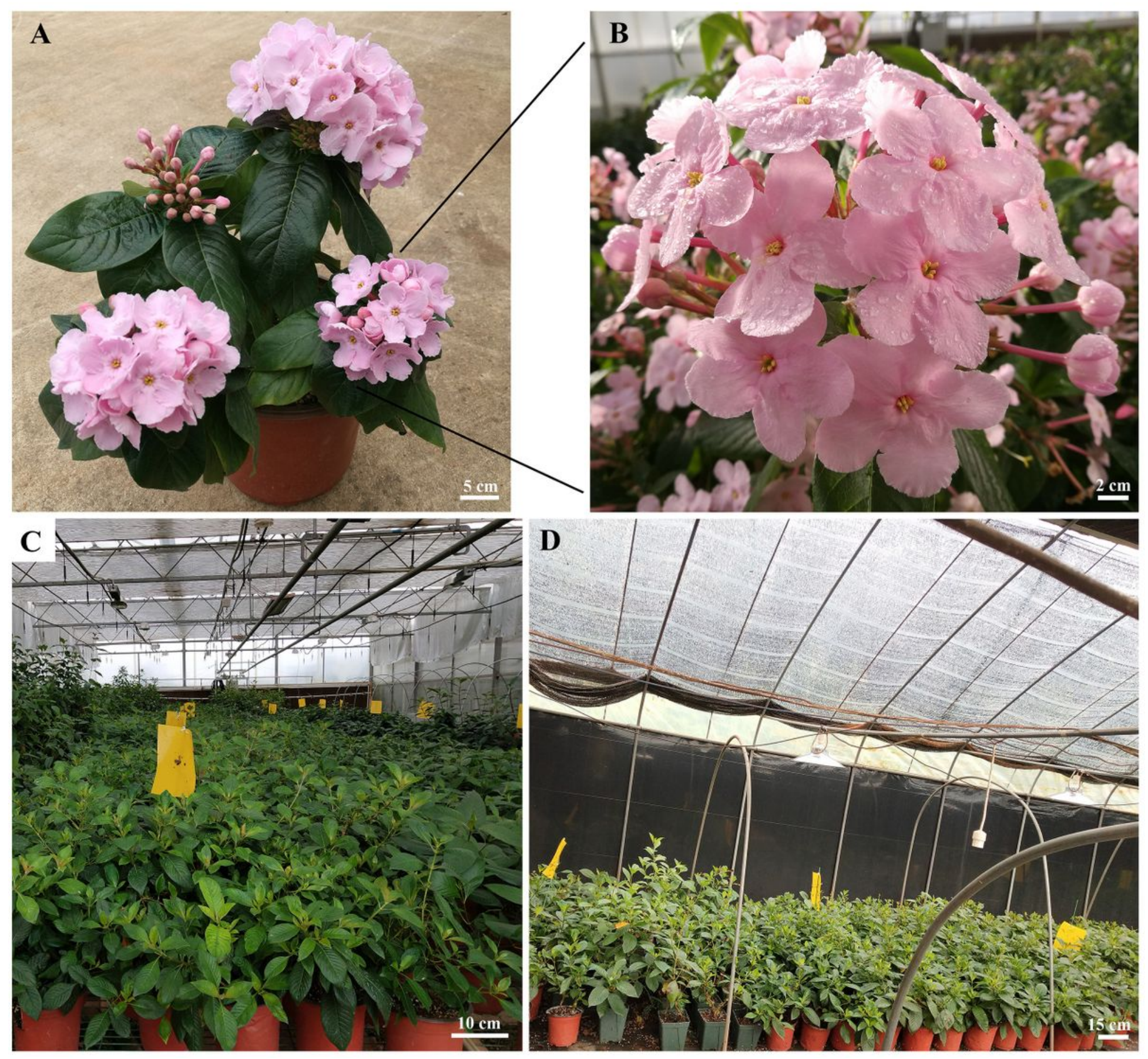

Figure 1

Features of Luculia gratissima 'Xiangfei' and the overview of greenhouses under two different photoperiods. a Whole plant of L. gratissima 'Xiangfei'. b Flowers of L. gratissima 'Xiangfei'. c Greenhouse under night-break treatment. $d$ Greenhouse under short-day photoperiod. 

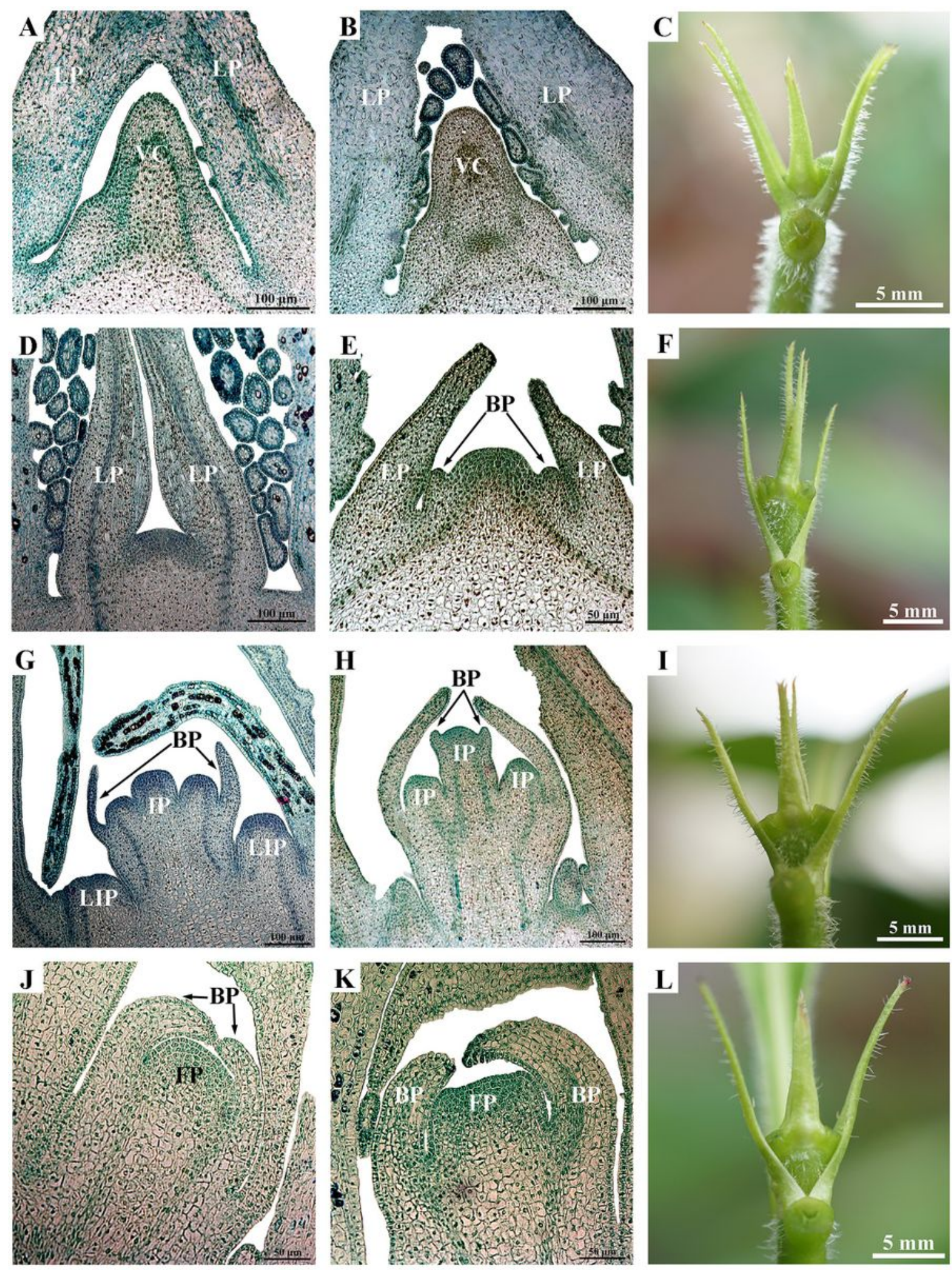

Figure 2

Luculia gratissima morphological and histological characteristics, shoot apexes at five time points upon short-day treatment. a-c Vegetative buds in the undifferentiated stage (SD0 to SD7). $d-f$ Bract primordial differentiation stage (SD10). a, b, d, g, h Histological images obtained from paraffin-embedded sectioned samples (scale bar: $100 \mu \mathrm{m}$ ). e, j, k Histological images obtained from paraffin-embedded sectioned samples (scale bar: $50 \mu \mathrm{m}$ ). c, f, i, I The external morphology of shoot apexes at different developmental 
stages (scale bar: $5 \mathrm{~mm}$ ). BP: bract primordia; FP: floret primordia; IP: inflorescence primordia; LIP: lateral inflorescence primordium; LP: leaf primordia; VC: vegetative cone.

Kruskal-Wallis H test
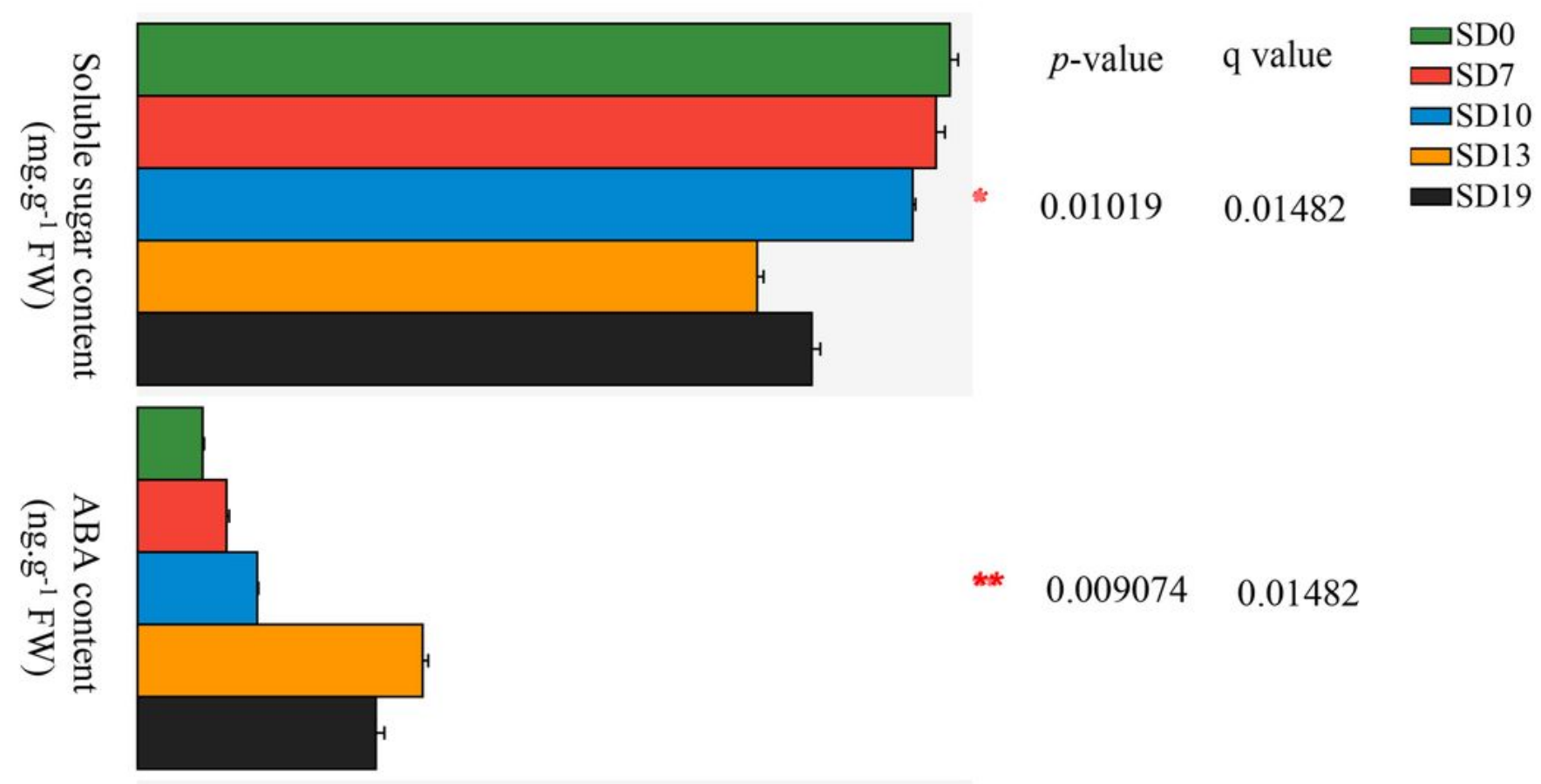

$$
\text { ** } 0.009074 \quad 0.01482
$$

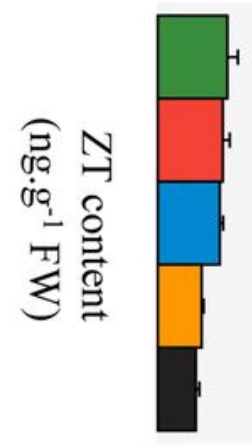

* 0.024060 .02406

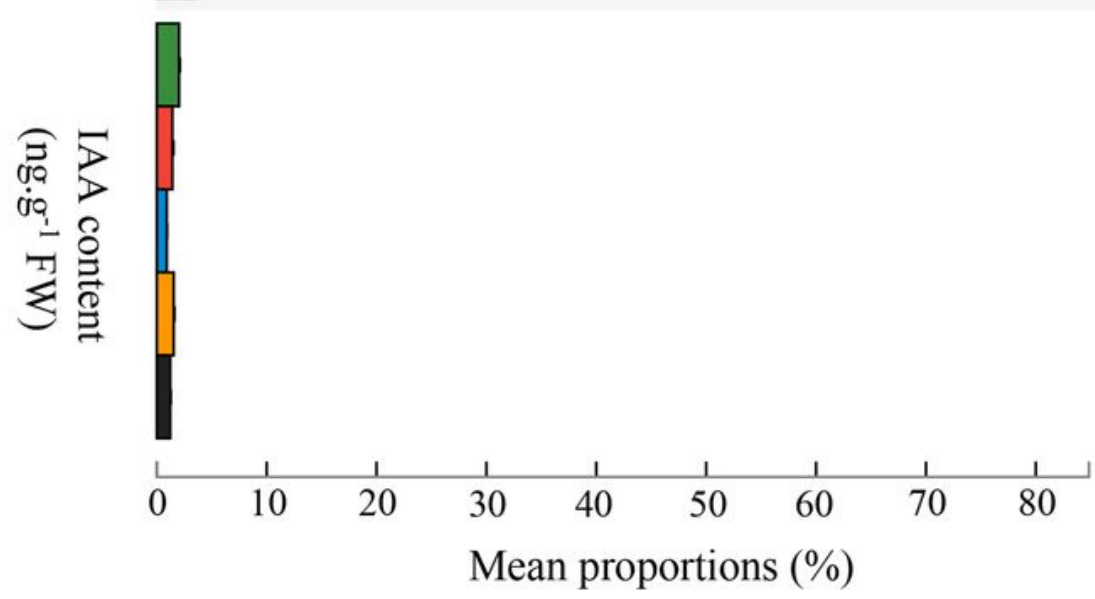

\section{Figure 3}

Luculia gratissima endogenous soluble sugar content and hormonal changes, shoot apexes at five stages upon short-day treatment. The $y$-axis shows soluble sugar and four hormones, and the $x$-axis 
shows the average relative abundance of the endogenous soluble sugars and hormones. Colored columns represent different developmental stages. $* 0.01<p \leq 0.05, * \star 0.001<p \leq 0.01$.
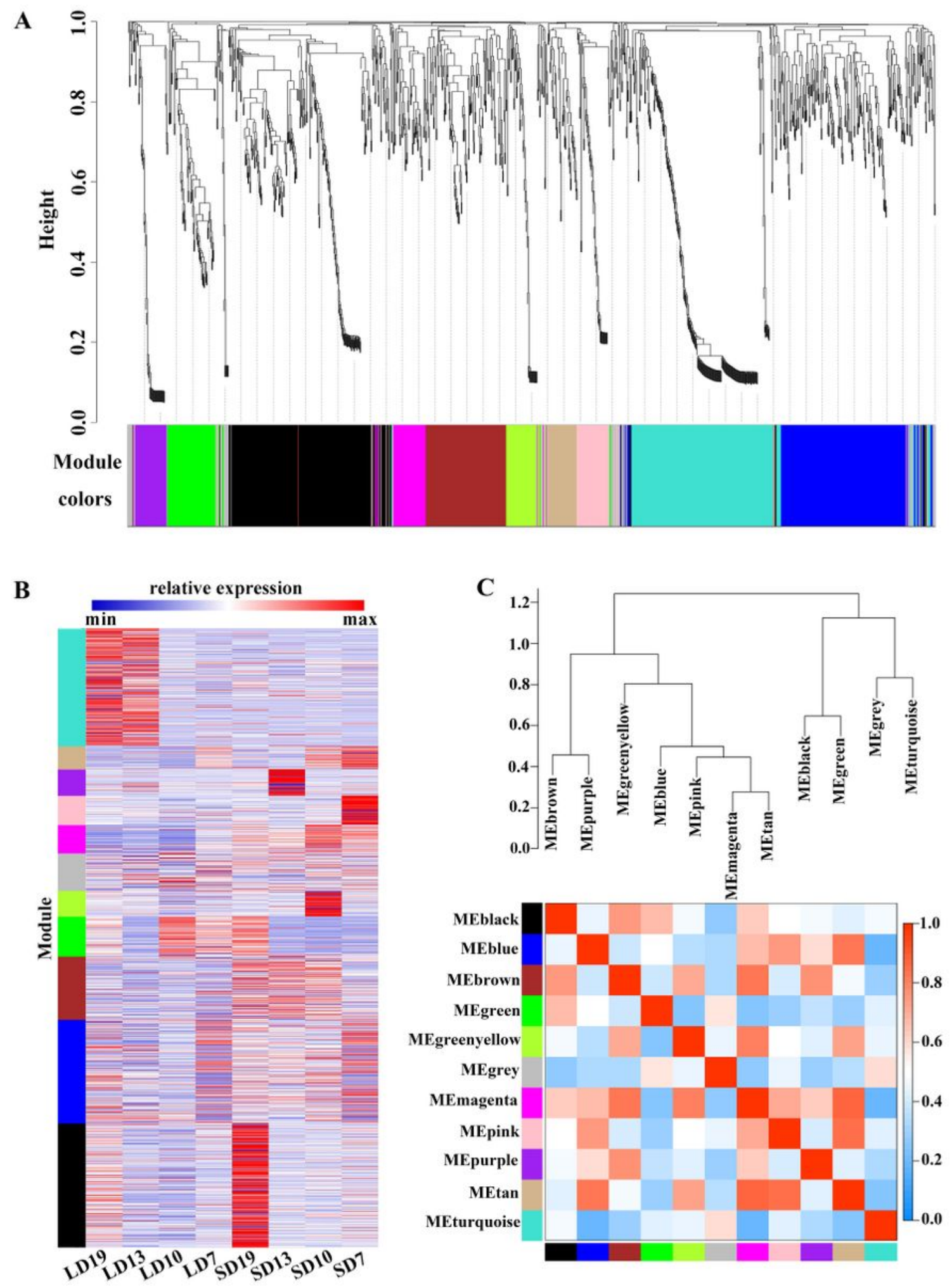

Figure 4

Weighted co-expression network analysis of 1,226 DEGs at four developmental stages of Luculia gratissima, short- or long-day treatments. a Hierarchical cluster tree showing the co-expression modules, with each tree leaf representing one gene. The major tree branches constitute 11 modules labelled by 
different colors. b Heat map of gene relative expression of different modules ( $y$-axis) in eight samples ( $x$ axis). The normalized RPKM value for an individual gene at a given developmental stage is indicated in a green (low expression) to red (high expression) scale. c Eigengene network representing the relationships among the modules and the clinical trait weight. The hierarchical clustering dendrogram of the eigengenes shows the relationships among the modules, whereas the heat map shows the correlation between the different modules, with deeper red color representing a stronger correlation.

relative expression
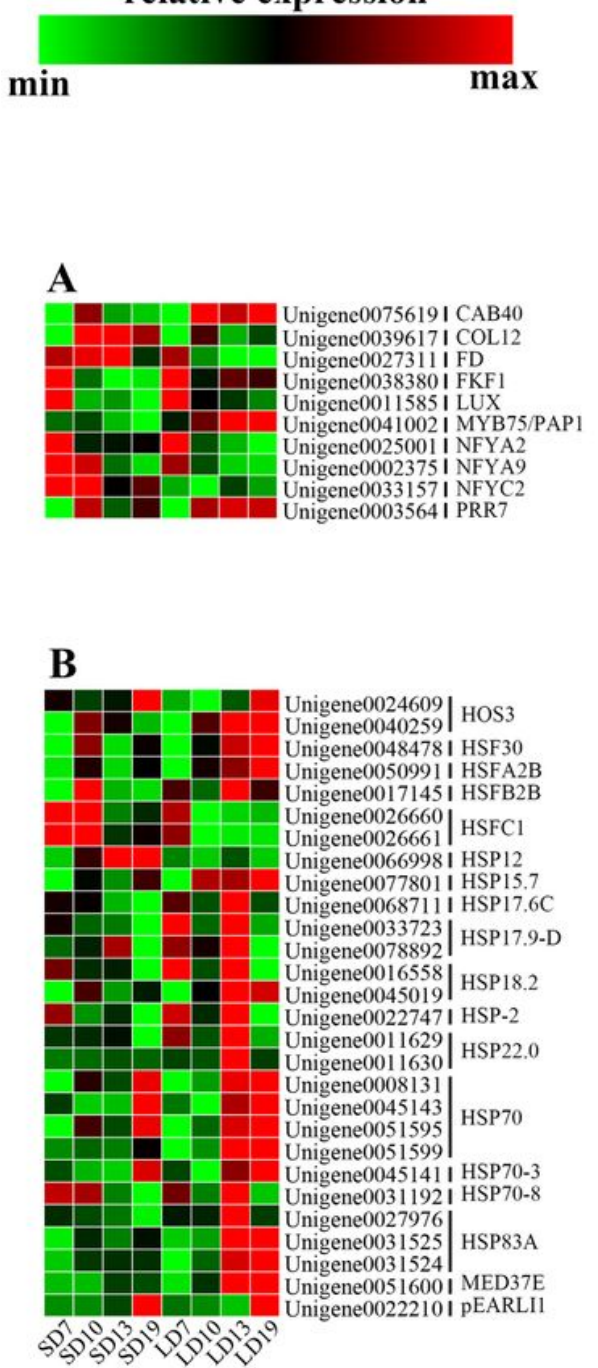

C

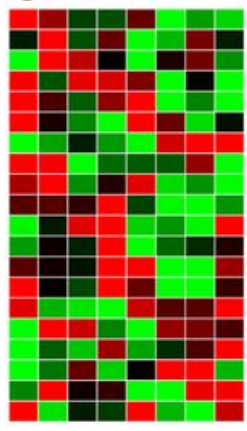

Unigene0034382 ICYP707A1 Unigene0042754 $\mid$ NCED1 Abscisic acid Unigene0035296IYUC4 I Auxin Unigene0036368 I CYP734A II Brassinolide Unigene0036368 1 CYP734 Unigene0036599 ICKX7
Unigene0029738 I CYP735A1
Unigene0033246I LOG3 Unigene0008758ILOG $\quad$ Cytokinin Unigene0073526 I LOG5 \begin{tabular}{l|l} 
Unigene0023297 & LOG8
\end{tabular} Unigene 00307321 GA2OXI \begin{tabular}{l|l|l} 
Unigene0073113 & GA2OX8 & Gibberellin \\
Unigene0007459 & GA2OX8
\end{tabular} $\begin{array}{ll}\text { Unigene0020912 I JMT } & \text { Jasmonic acid } \\ \text { Unigene0004033 I UGT74FI } & \text { ISalicylic acid }\end{array}$ nigene0069349 UCT74FI ISalicylic acid Unigene0018504 CCD7 IStrigolactone \begin{tabular}{l|l} 
Unigene0050952 & ZOG1 $\quad$ Zeatin
\end{tabular}

\section{D}

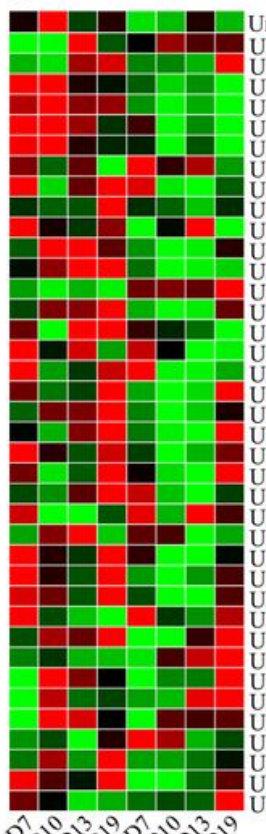

किषे
E

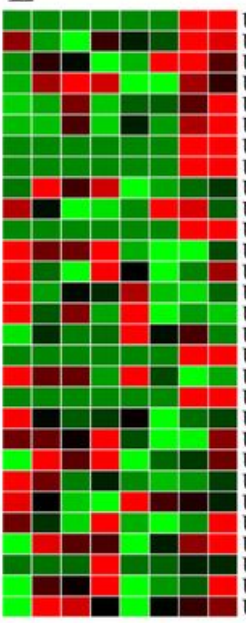

Unigene0076531 I AKR 1B 1 Unigene0035031 I BAM3 Unigene 00130881 BGLU2 Unigene0006889 Unigene0006889 $\mid$ BACOVA_02659 Unigene0032743 \begin{tabular}{l|l} 
Unigene0011084 & ENO1
\end{tabular} Unigene00102961 GAE2 Unigene 0055621 I At $5 \mathrm{~g} 18200$ Unigene $00058461 \mathrm{GAPDH}$ Unigene0036240 $\mid$ GOLS2 Unigene0009721 GOLS2 Unigene0044869 HK Unigene 0031285 I INVA Unigene0009368 I LDHA Unigene0049182 I PGDH2 Unigene0073914 I PKM Unigene0041893 I RFS Unigene0007961 |SGR | Unigene0035765 0012661 I SWEET 16 Unigene 00135551 TPS 10 Unigene 00546041 TPS 11 Unigene0004913 I TPS13 \begin{tabular}{l|l|l|} 
Unigene0062998 & TPS31 \\
Unigene0019787 & TPS7
\end{tabular} \begin{tabular}{l|l} 
Unigene0019787 & TPS7 \\
Unigene0024389 & TPS
\end{tabular}

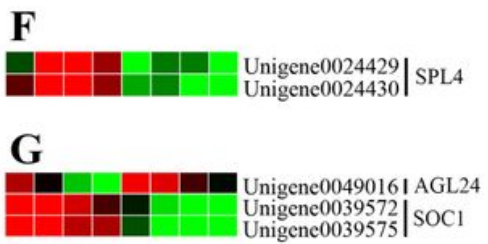

H

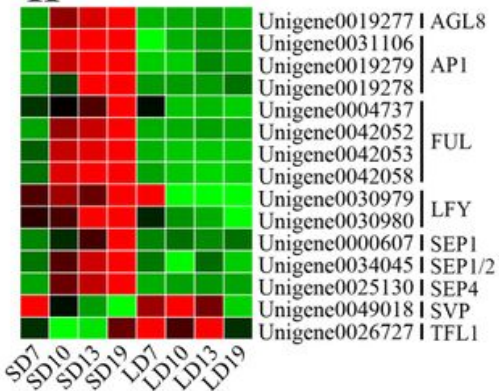

\section{Figure 5}

Expression profiles of genes associated with Luculia gratissima floral transition at four developmental stages, short- or long-day treatments. Relative expression profile of (a) photoperiod pathway-related genes, (b) ambient temperature pathway-related genes, (c) phytohormone metabolism-related genes, (d) phytohormone signal transduction-related genes, (e) sugar signal-related genes, (f) aging pathway-related genes, (g) floral integrator-related genes, and $(\mathrm{h})$ floral meristem identity genes. The normalized RPKM value for an individual gene at a given developmental stage is represented in a green (low expression) to red (high expression) scale. 


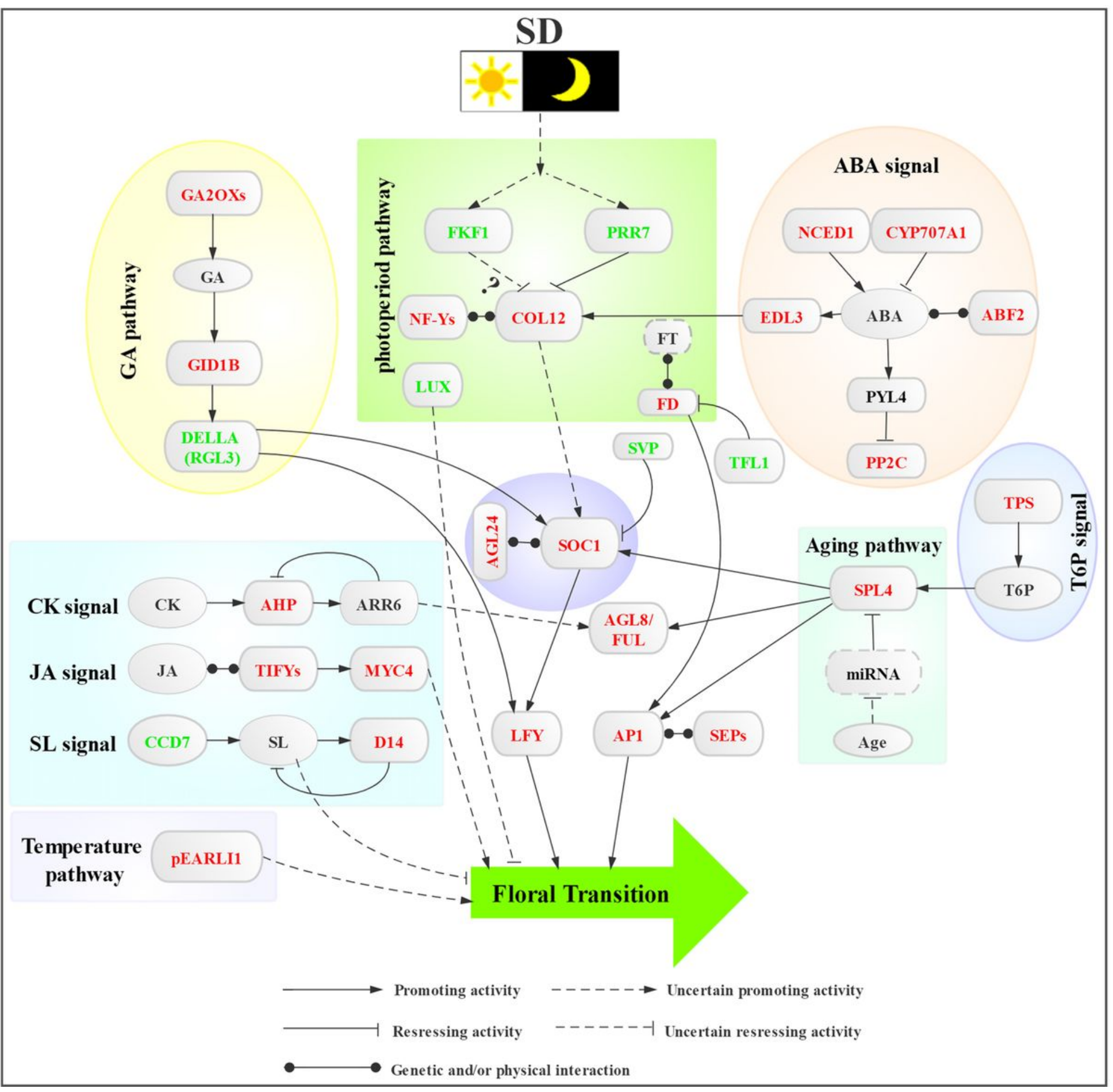

Figure 6

Proposed regulatory network of short-day photoperiod-induced floral transition in Luculia gratissima. Colored fonts represent down-regulated (green) or up-regulated (red) genes.

\section{Supplementary Files}

This is a list of supplementary files associated with this preprint. Click to download. 
- Additionalfile17Tables9.docx

- Additionalfile13Tables7.xlsx

- Additionalfile12TableS6.xlsx

- Additionalfile15Fig.S7.docx

- Additionalfile14TableS8.xlsx

- Additionalfile11TableS5.xlsx

- Additionalfile10Fig.S6.docx

- Additionalfile9Fig.S5.docx

- Additionalfile8Fig.S4.docx

- Additionalfile7TableS4.xlsx

- Additionalfile16SupplementaryData.docx

- Additionalfile6TableS3.xIsx

- Additionalfile5Fig.S3.docx

- Additionalfile4Fig.S2.docx

- Additionalfile3Fig.S1.docx

- Additionalfile2TableS2.docx

- Additionalfile1TableS1.docx 